\title{
Nuevos aspectos en el estudio de la partícula D en el experimento FOCUS de Fermilab
}

\author{
por: \\ José A. Quiñones González \\ Tesis sometida en cumplimiento parcial \\ de los requisitos para el grado de \\ MAESTRÍA EN CIENCIA \\ en \\ Física \\ UNIVERSIDAD DE PUERTO RICO \\ RECINTO UNIVERSITARIO DE MAYAGÜEZ, \\ 2005
}

Aprobada por:

Ángel López Berríos, Ph.D.

Fecha

Presidente, Comité Graduado

Fecha

Miembro, del Comité Graduado

Rubén Méndez, Ph.D.

Fecha

Miembro, Comité Graduado

Fecha

Omar Colón, Ph.D.

Representante de la Escuela Graduada

Héctor J. Jiménez, PhD

Fecha

Director del Departamento

José Mari Mutt, $\mathrm{PhD}$

Fecha

Director de la Escuela Graduada 


\begin{abstract}
The purpose of this work is to improve the reconstruction techniques of the decays of the particles that contain charm in the quark composition using the information of the Target Silicon Detector of the experiment E831 (FOCUS). That experiment runs during 1997 to 1998 in Fermilab National Laboratory. The objective of the experiment was improving the understanding of the particles that contain charm. Adding the Target Silicon Detector information in the reconstruction process of the primary vertex the position error. This reduction produces an improvement in the mass signal and the knowledge of the charm particles properties. This ad to the possibility's that in other analysis will use the techniques developed in this work.
\end{abstract}




\section{Resumen}

El propósito de este trabajo es mejorar las técnicas de reconstrucción de los decaimientos que contienen el quark "charm" utilizando la información del detector de silicio del blanco del experimento E831 (FOCUS). Dicho experimento se llevó a cabo en los años 1997 al 1998 en el laboratorio nacional de Fermilab. El propósito del experimento era superar a los experimentos anteriores hechos sobre el quark charm. Al añadir la información del detector de silicio lo que se desea es reducir el error en la determinación de la posición del vértice primario o vértice de producción de la partícula de estudio. Esta disminución de error repercute en el mejoramiento de la señal de masa y la determinación de otras propiedades de la partícula que contienen charm y la posibilidad de que en estudios posteriores las técnicas desarrolladas puedan ser utilizadas en otros trabajos. 


\section{Dedicatoria}

A mi esposa que amo con todo mi corazón Lina,

a Linita que es mi segunda hija y a mi pequeña Melanie. 


\section{Agradecimientos}

Agradezco al Divino Hacedor y Arquitecto del Universo, si es que existe y nos escucha. A mis padres, José A. Quiñones López mi padre que se ha ido a morar a la posada eterna donde ya las tormentas de la vida no alcanzaran; y a mi madre Bonifacia González Morales la cual me ayudo mucho con su apoyo y consejos. A mi abuela Catalina Morales Cordero que junto a mi padre partieron durante el tiempo en que se realizaba este trabajo a la posada eterna. A mi hijo Antonio Emil Quiñones que es mi alegría y esperanza. A el amor de mi vida Lina Soraya Llanos que llego de lejos para llenar mi vida de esperanza de nuevo con su presencia y que me dio a mi hija Melanie Sophia y a Linita. A mis compañeros de trabajo de la actualiza y del pasado; Alejandro Mirles, Carlos Rivera, Eduardo Ramirez que me ayudo mucho y me dio ideas útiles, Salvador Carrillo, Faviola Vázquez, Kennie Cruz, Alexis París, Eduardo Luiggi, y Carlos Pérez. A mi consejero Ángel López Berrios por su paciencia, al profesor Héctor Méndez por sus consejos y a Will E. Johns quien fue el que dio la idea de este trabajo. 


\section{Tabla de contenido}

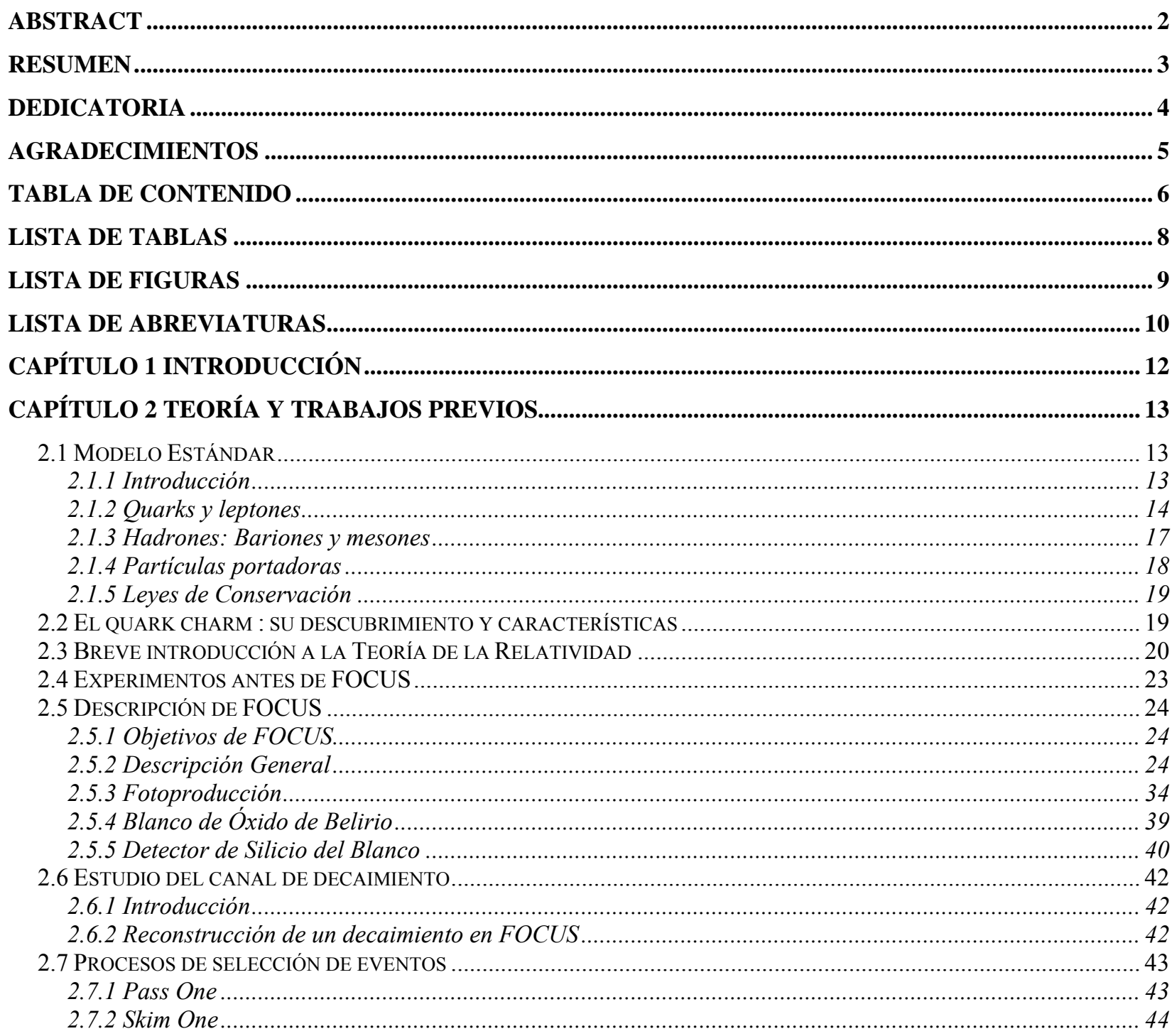

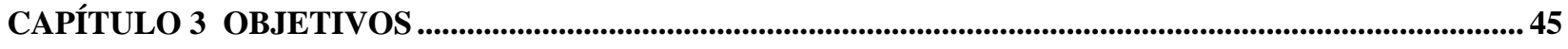

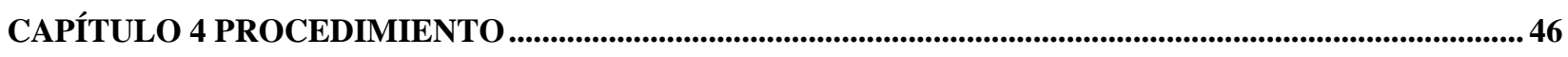

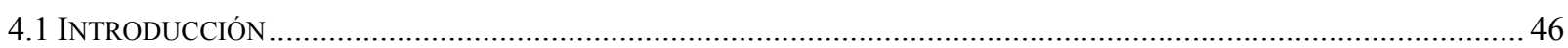

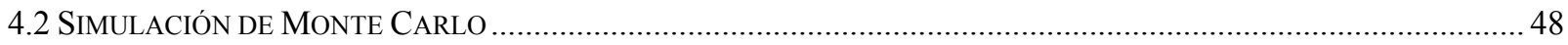

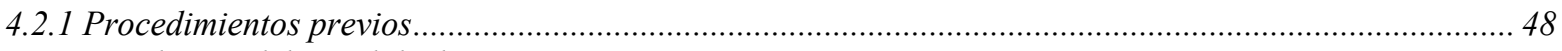

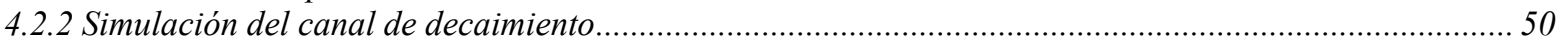

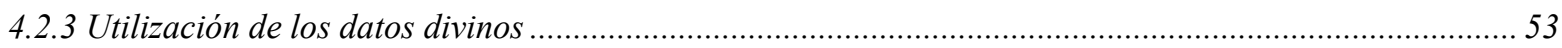

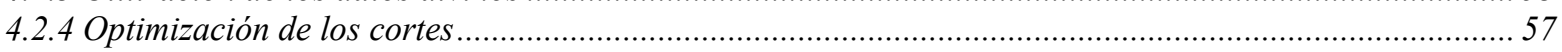

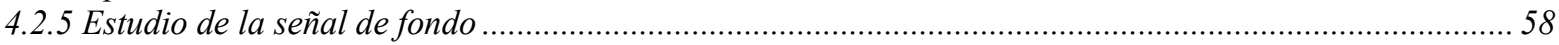

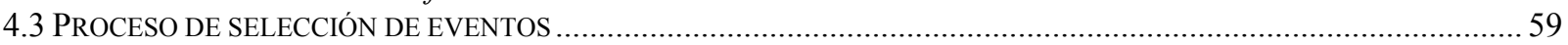

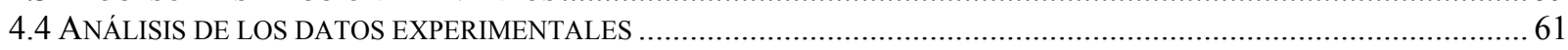

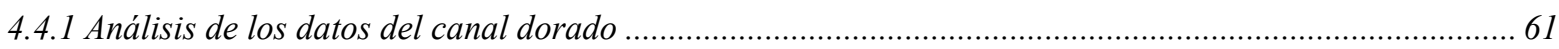

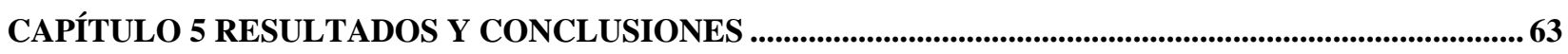

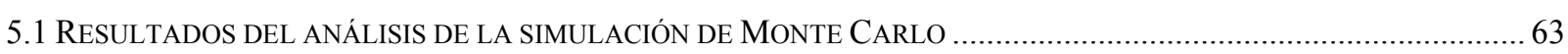

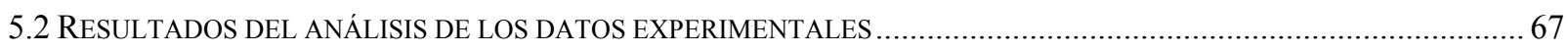

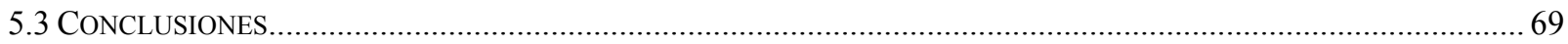

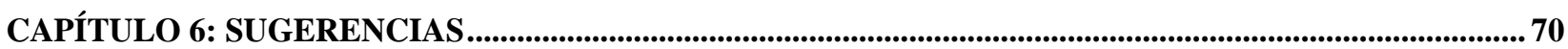




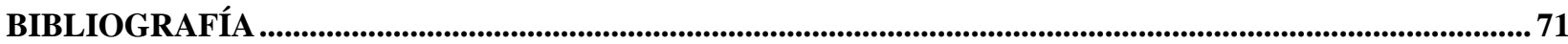




\section{Lista de Tablas}

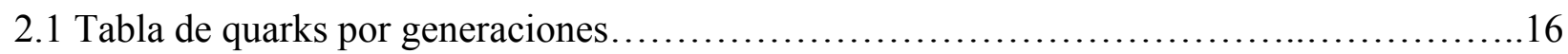

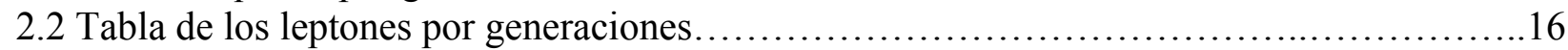

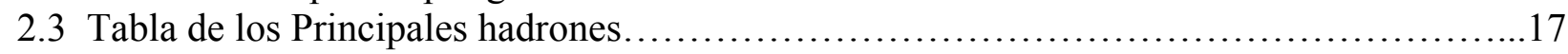

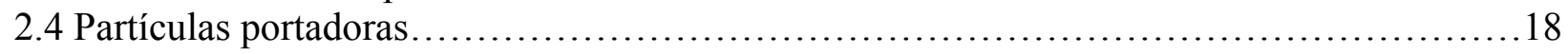

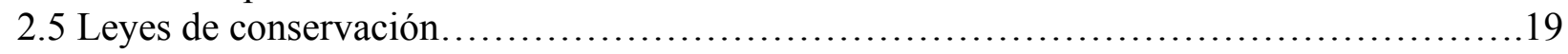

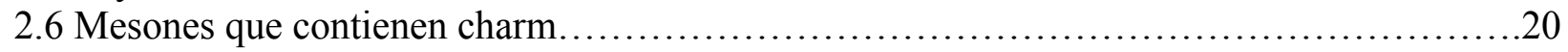

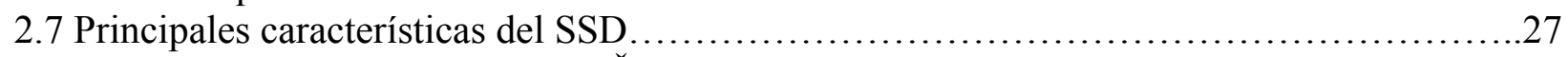

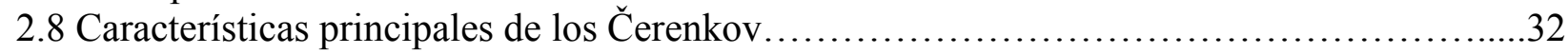

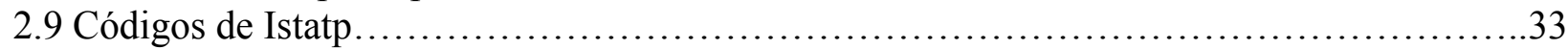

2.10 Descripción de la etapas de la producción del rayo de fotones..............................38

2.11 Datos de la geometría del área del blanco por periodos..................................40

2.12 Descripción de los diferentes "superstreams"......................................4 44

4.1 Información sobre las partículas principales candidatas para el estudio que contienen

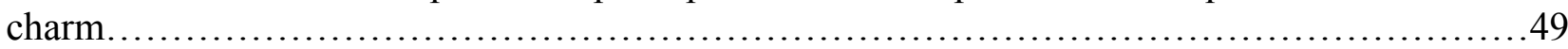

4.2 Razones de decaimiento para los diferentes canales de decaimiento de $\mathrm{D}^{+} \ldots \ldots \ldots \ldots \ldots \ldots \ldots \ldots . . . .50$

4.3 Variables que se guardan en cada determinación de un vértice primario.....................52

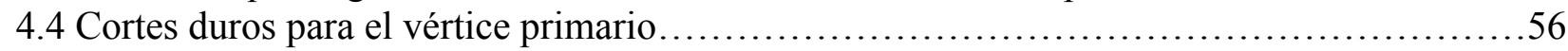

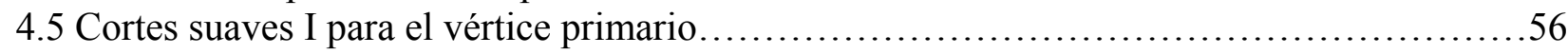

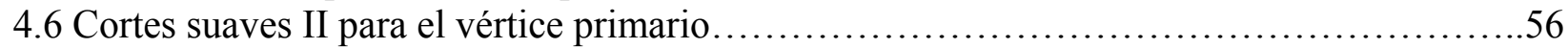

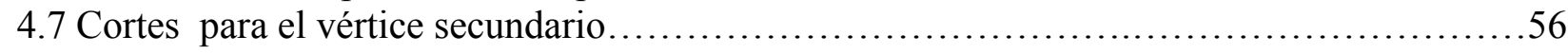

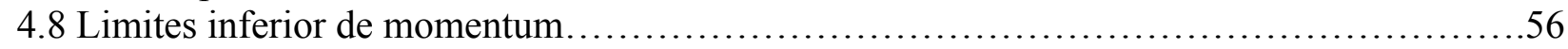

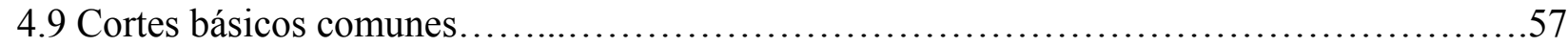

4.10 Cortes de selección utilizando en la primera fase de selección.............................60

4.11 Cortes de selección utilizados en la segunda fase de selección..............................60 


\section{Lista de Figuras}

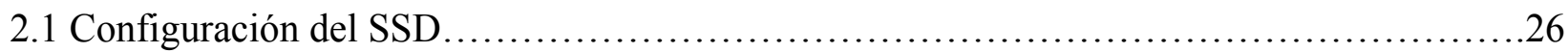

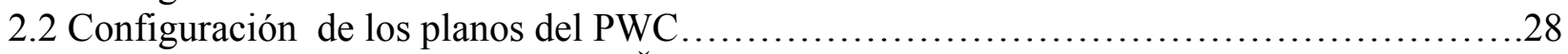

2.3 Emisión de la producción de la luz Čerenkov..........................................29

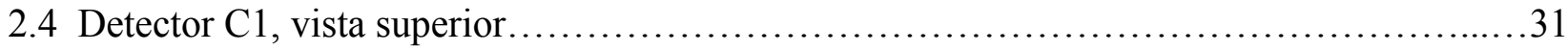

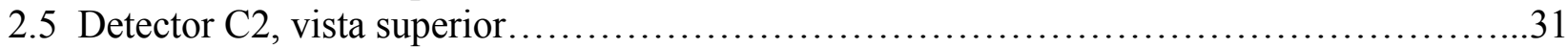

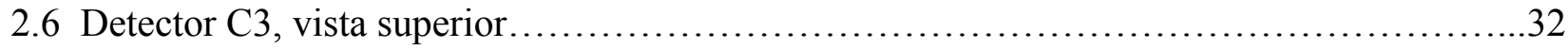

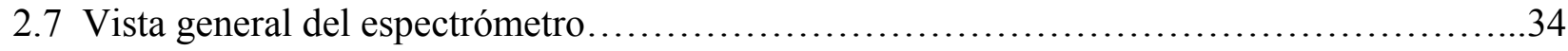

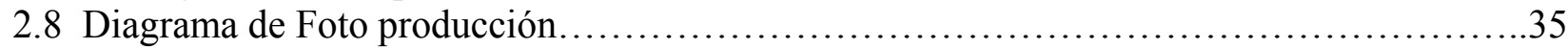

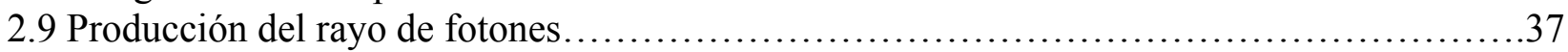

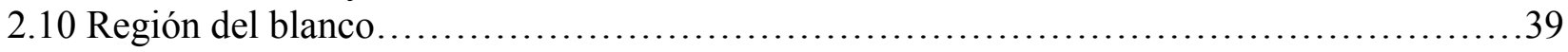

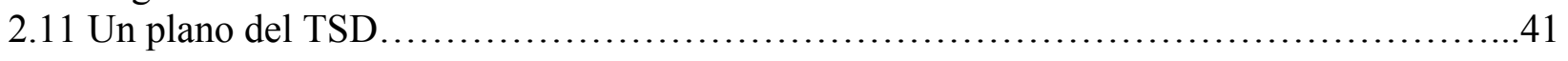

4.1 Diagrama de Feyman para el decaimiento $D^{+} \rightarrow K^{-} \pi^{+} \pi^{+} \ldots \ldots \ldots \ldots \ldots \ldots \ldots \ldots \ldots \ldots \ldots . \ldots 47$

4.2 Muestra de la estructura de una señal con ruido.......................................58

5.1 Eficiencias para los cortes suaves para los diferentes método de recontracción..............63

5.2 Eficiencias para los cortes en los que se utilizo la información del TSD...................64

5.3 Señal de masa reconstruida con la información del TSD ...............................65

5.4 Diferencia promedio para la posición del vértice primario de los datos reconstruidos y los

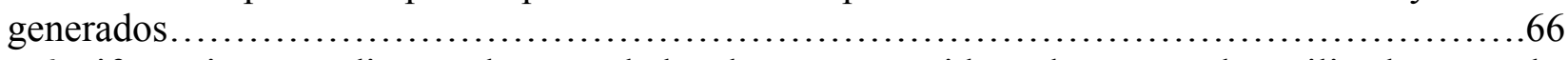

5.6 Diferencia promedio para la masa de los datos recontruidos y los generados utilizado Dvnucl

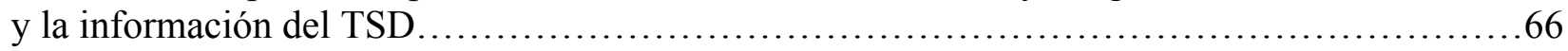

5.7 Rendimiento versus cortes para los cortes suaves....................................67

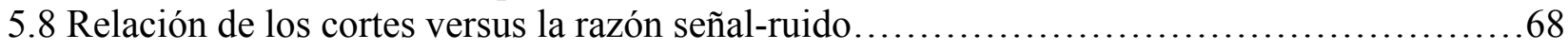

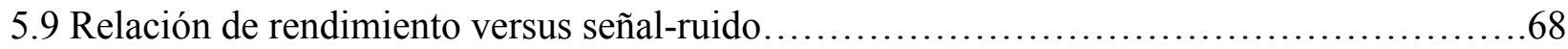




\section{Lista de Abreviaturas}

$\mathrm{CP}$

DVFITSD

DVFREE

DVNUCL

E831

FOCUS

$\mathrm{Gb}$

GD

$\mathrm{GeV}$

ISO1

ISO2

Istatp

$l / \sigma$

$\mathrm{MC}$

$\mathrm{MeV}$

$\mathrm{N}_{\text {gen }}$

$\mathrm{N}_{\text {obs }}$
"Charge Parity"

Rutina que añade la información del TSD

reconstrucción

Rutina de reconstrucción del vértice primario

Rutina de recontracción del vértice primario

Código del experimento

"Fotoproduction of Charm with Upgraded

Spectometer"

Gigabyte

"God Data"

Giga electrón voltios

Corte de insolación 1

Corte de insolación 2

Codigo de los detectores de Cerenkov

"l over sigma"

Monte Carlo

Mega electrón voltios

Número de eventos generados

Número de eventos observados 
PCL

$\mathrm{P}_{\mathrm{D}}$

PWC

QCD

QED

$\mathrm{RD}$

SCL

SSD

$\mathrm{Tb}$

TSD

$Z_{\text {pri }}$

$\mathrm{Z}_{\mathrm{sec}}$
Nivel de confianza del vértice primario

Momentum de la partícula D

"Propotional Wire Chamber"

"Quantum Chromodynamic"

"Quantum Electrodynamic"

"Recostructed Data”

Nivel de confianza del vértice secundario

Detectores de planos

Terabyte

"Target Silicon Detector"

Posición en el eje de $\mathrm{z}$ del vértice primario

Posición en el eje de $\mathrm{z}$ del vértice secundario 


\section{Capítulo 1 Introducción}

El propósito de este trabajo es mejorar la precisión en la reconstrucción de los vértices de decaimientos de las partículas que contengan charm. Para poder lograr nuestros objetivos se utilizó la información del detector que se encontraba más cerca del punto de producción de las partículas con charm. Este detector se llamo el Detector de Silicio del Blanco ("Target Silicon Detector" en ingles). Las partículas que contienen charm de mayor vida viajan aproximadamente $1.5 \mathrm{~cm}$. en el experimento. A velocidades relativistas esta distancia es recorrida en un tiempo de $5 \times 10^{-11}$ segundos aproximadamente. Por consiguiente se necesitaba un detector que estuviera bien cerca del blanco para que las partículas con charm pudieran detectarse de forma directa. En comparación con los pasados experimentos que estudiaron el quark charm, FOCUS poseían una gran ventaja no solo en su estadística sino también en la resolución por la utilización de este detector. El trabajo se concentra en poder desarrollar y probar las técnicas de reconstrucción para este y posibles futuros experimentos que posean detectores similares. 


\section{Capítulo 2 Teoría y trabajos previos}

"Quien no se impresione de la Mecánica Cuántica es que no la entiende."

Niels Bohr

Físico danés

\subsection{Modelo Estándar}

\subsubsection{Introducción}

El Modelo Estándar es la teoría que modela la composición de la materia a niveles subatómicos y las interacciones que ocurren entre las partículas elementales, que son las constituyentes de todo a este nivel. Estas interacciones son el producto de la existencia de las fuerzas fundamentales [1]. Al día de hoy la evidencia experimental demuestra que existen cuatro fuerzas fundamentales, de las cuales tres están contenidos o modelados en el Modelo Estándar. Las fuerzas fundamentales son la gravedad, electromagnetismo, la fuerza nuclear débil y las fuerza de color. Hasta el día de hoy no existe una teoría a nivel cuántico que explique la gravedad y por dicha razón no se encuentra contenida en el Modelo Estándar. Esto se debe a una serie de incompatibilidades que existen entre la teoría de la Relatividad y la Mecánica Cuántica [2,3]. Desde la perspectiva histórica la fuerza de gravedad fue la primera que el hombre tuvo conocimiento de su existencia. El primer acercamiento para lograr un entendimiento científico fueron los trabajos realizados por Galileo Galilei y Isaac Newton [4], claro esto desde una perspectiva mas cercana al rigor científico actual. Luego los trabajos de Albert Eistein [5] a principios del siglo XX son el punto de partida de la perspectiva actual. La fuerza de gravedad actúa sobre toda partícula.

La primera fuerza que se encuentra incluida en el Modelo Estándar es la fuerza electromagnética. Esta es responsable de las interacciones entre las partículas con carga eléctrica. En un principio se pensaba que la electricidad y el magnetismo eran dos fenómenos diferentes por consiguientes que no tenían un origen en común. Fue por el trabajo realizado por James Clarck Maxwell durante la segunda mitad del siglo XIX [6], que se logro comprender que la electricidad y el magnetismo eran dos manifestaciones diferentes de las misma fuerza. Esta fue la primera unificación que se realizó. Una unificación es cuando se logra estructurar una teoría con la cual podemos lograr comprender varios fenómenos diferentes. En la actualidad la comprensión de la fuerza electromagnética ha llegado a niveles quánticos. La teoría que nos ayuda a hacer esto es la que se conoce como Electrodinámica Cuántica o QED [7] por sus siglas en ingles. La misma fue 
desarrollada a mediados del siglo XX y es una de las partes fundamentales del Modelo Estándar.

Las otras dos fuerzas incluidas en el Modelo solo se manifiestan a nivel subatómico, son la fuerza nuclear débil y la fuerza de color. Las mismas fueron descubiertas durante las primeras décadas del siglo XX. El radio de acción de la fuerza débil es de aproximadamente $10^{-12} \mathrm{~m}$ y el de la fuerza de color de aproximadamente $10^{-18} \mathrm{~m}$ [8]. La fuerza débil es responsable del decaimiento beta y de los decaimientos en los cuales un quark cambia de "sabor". La fuerza de color es la responsable de mantener unidos los quarks para formar otras partículas. La fuerza nuclear fuerte es una interacción residual de la fuerza de color y es la responsable de mantener unidos los protones y neutrones en el núcleo.

Como cualquier otro desarrollo humano el Modelo Estándar es el producto de muchos años de trabajo, los cuales comprenden desde comienzos del siglo XX con los primeros trabajos de Mecánica Cuántica hasta nuestros días. El punto más neurálgico fue la teoría propuesta por Gell-Mann y Zweig, la teoría de los quarks [9]. Durante la década de los cincuenta y comienzos de los sesentas se descubrieron un gran número de partículas, y no se podía determinar una relación entre ellas. La teoría de los quarks no solo nos brindo de las herramientas para poder clasificar y comprender los mecanismos que actuaban en las partículas que hasta el momento se conocían, sino también se pudieron hacer predicciones las cuales luego fueron verificada, lo cual le dio mas validez a la teoría.

\subsubsection{Quarks y leptones}

Las partículas fundamentales que se conocen hasta el día de hoy se pueden dividir en tres grupos, los quarks, los leptones y los portadores de fuerza o bosones. Para que una partícula sea considera fundamental si cumple con las siguientes dos condiciones. La primera de las condiciones se conoce como la condición de Demócrito ${ }^{1}$, esta dice que no puede tener estructura interna y por ende no posee componentes. La segunda condición es la de Boscovich², la cual expone que la partícula es puntiforme, esto quiere decir que no tiene volumen. Estas dos condiciones las cumplen las partículas que pertenecen a los grupos antes mencionados [10].

El primer grupo de partículas fundamentales esta constituido por los quarks. Al

\footnotetext{
${ }^{1}$ Filosofo griego que fue el primero en proponer la existencia de los átomo. La palabra átomo quiere decir sin división en griego.

${ }^{2}$ Matemático ruso del siglo 18, desarrollo una teoría atómica partiendo de las leyes de Newton.
} 
momento se conocen seis tipos o sabores de quarks, los cuales se agrupan en tres generaciones. Una generación de partículas fundamentales esta compuesta de dos quarks y dos leptones. Los quarks poseen varias características partículares. Los quarks nunca están en la naturaleza en estado libre, es decir, estos siempre están unidos formando dúos o tríos. Al unirse forman otras partículas, las cuales conocemos como hadrones, la palabra hadrón proviene del griego y quiere decir duro. Los hadrones a su vez se agrupan en dos grupos: los mesones y los bariones. Para realizar esta clasificación se utiliza como criterio el número de quarks que constituyen al hadrón. De ser una pareja, esta estará formada por un quark y un anti-quark, a este tipo de partícula se le conoce como un mesón. Por otro lado si es un trío de quarks o de anti-quarks a la partícula se le conoce como un barión. Como antes habíamos mencionando, la existencia de los quarks fue propuesta por Gell-Mann y Zweig. Esta teoría resolvió el problema que representaba la evidencia experimental de a principios de los sesentas. Existía evidencia de que un gran número de las partículas descubiertas hasta ese momento tenia estructura interna.

La propuesta de Gell-Mann y Zweig era que existían tres partículas las cuales al combinarse formaban las partículas que hasta ese momento se habían detectado, exceptuando al electrón, muón y sus neutrinos correspondientes. Posteriormente a estas partículas se le llamo up, down y strange. Un dato curioso es que Gell-Mann los llamo quarks y que Zweig los llamo “aces", pero el nombre de quarks prevaleció. Entre las características principales de los quarks están, que tienen carga eléctrica fraccionada, su cargas son $+2 / 3$ e y $-1 / 3$ e. Esto para el caso de los quarks, si son anti-quarks las cargas tienen signos contrarios. Otra de las características es que son fermiones. La forma más sencilla de dividir las partículas es en términos de la distribución a la que obedecen. Existen dos distribuciones las Fermi-Dirac y la Bose-Eistein [11]. Si el número de espín de la particula es fraccionario la distribución que obedece es la de Fermi-Dirac. Si el valor es un número entero la distribución que obedece es la de Bose-Eistein.

Los leptones es el siguiente grupo de partículas fundamentales. En cada generación tenemos dos leptones lo que al final da un total de seis leptones conocidos, con sus correspondientes antipartículas [12]. El nombre de leptón proviene del griego y quiere decir pequeño o liviano. Estas partículas tienen espines de $1 / 2$ lo que hace que se tengan que clasificar como fermiones. Si el leptón tiene carga eléctrica, esta será entera. Para los de materia es -1 y para los de antimateria es +1 . Solo tres leptones poseen carga eléctrica, estos son el electrón, muón y tau. Los demás, llamados neutrinos, no tienen carga eléctrica y de acuerdo a la reciente evidencia experimental, poseen una masa bien pequeña, de un orden menor a $10 \mathrm{eV}$. Estas características hacen que los neutrinos solo interactúen a través de la fuerza nuclear débil. En 
cambio los que tienen carga eléctrica interactúan por la fuerza nuclear débil y por la electromagnética. Los leptones se pueden encontrar en estado libre.

En las siguientes dos tablas presentamos los diferentes quarks y leptones. Las tablas están divididas por generación. Existen tres generaciones en el Modelo Estándar, esto no lleva a tener 12 partículas fundamentales, esto sin incluir la antimateria y la carga de color.

Tabla 2.1

Tabla de los quarks por generación

\begin{tabular}{|l|l|l|}
\hline \multicolumn{1}{|c|}{ Generación I } & \multicolumn{1}{|c|}{ Generación II } & \multicolumn{1}{c|}{ Generación III } \\
\hline u (up) & c (charm) & t (top) \\
carga: $+2 / 3 e$ & carga: $+2 / 3 e$ & carga: $+2 / 3 e$ \\
masa: $310 \mathrm{MeV} / \mathrm{c}^{2}$ & masa: $1500 \mathrm{MeV} / \mathrm{c}^{2}$ & masa: $22500 \mathrm{MeV} / \mathrm{c}^{2}$ \\
\hline $\begin{array}{l}\text { d (down) } \\
\text { carga: }-1 / 3 e\end{array}$ & s (strange) & $\mathbf{b}$ (bottom) \\
masa: $310 \mathrm{MeV} / \mathrm{c}^{2}$ & masa: $505 \mathrm{MeV} / \mathrm{c}^{2}$ & carga: $-1 / 3 e$ \\
masa: $5000 \mathrm{MeV} / \mathrm{c}^{2}$
\end{tabular}

Tabla 2.2

Tabla de los leptones por generación

\begin{tabular}{|c|c|c|}
\hline Generación I & Generación II & Generación III \\
\hline $\begin{array}{l}\mathbf{e} \\
\text { carga: }-1 e \\
\text { masa: } 0.511 \mathrm{MeV} / \mathrm{c}^{2}\end{array}$ & $\begin{array}{l}\mu \\
\text { carga: }-1 e \\
\text { masa: } 106.6 \mathrm{MeV} / \mathrm{c}^{2}\end{array}$ & $\begin{array}{l}\tau \\
\text { carga: }-1 e \\
\text { masa: } 1784 \mathrm{MeV} / \mathrm{c}^{2}\end{array}$ \\
\hline $\begin{array}{l}v_{e} \\
\text { carga: } 0 \\
\text { masa: } \approx 0 \mathrm{MeV} / \mathrm{c}^{2}\end{array}$ & $\begin{array}{l}v_{\mu} \\
\text { carga: } 0 \\
\text { masa: } \approx 0 \mathrm{MeV} / \mathrm{c}^{2}\end{array}$ & $\begin{array}{l}v_{\tau} \\
\text { carga: } 0 \\
\text { masa: } \approx 0 \mathrm{MeV} / \mathrm{c}^{2}\end{array}$ \\
\hline
\end{tabular}




\subsubsection{Hadrones: Bariones y mesones}

Como mencionamos en la parte anterior los quarks se agrupan en pares o tríos, y de esta forma es que forman los hadrones. A su vez los hadrones se dividen en dos grupos; los mesones y los bariones [13]. El primer grupo son los mesones. Estos están formados por un quark y un antiquark, tienen interacciones mediante la fuerza débil, fuerte y electromagnética si poseen carga eléctrica. El espín de los mesones es entero. Esto quiere decir que obedecen la estadística Bose-Eistein. Los piones y kaones son ejemplos de los mesones. Un dato importante es que tiene estados excitados. Estos no llevan a tener dos o más mesones con la misma composición de quarks pero diferentes masas. Esto se debe que la diferencia de energía de un sistema y el otro produce un cambio en la masa de las partículas muy significativo. A estos estados se le llaman estados exitados.

El otro grupo de hadrones es el de los bariones. Estos se componen de tres quarks o tres antiquarks. Los espines de los bariones son fracciónales, hace que obedezcan la estadística Fermi-Dirac. Un importante número cuántico de los bariones es el llamado número barionico. El mismo se tiene que conservar en todos los procesos. Si un barión decae entre las partículas hijas deber haber un barión. Un dato importante son las investigaciones de los pasados años sobre el decaimiento del protón, los resultados apuntan que su vida media es del orden de la edad del Universo o mayor. El número baríonico es representado por la letra $\mathrm{B}$. Los bariones tienen $\mathrm{B}=1$ y los antibariones es $\mathrm{B}=-1 \mathrm{y}$ las temas partículas $\mathrm{B}=0$. Todos los bariones tienen masas mayores que la del protón, lo que no lleva a que el no podría decaer sin violar el número baríonico. En la siguiente tabla están algunos de los hadrones más comunes en la física de partículas.

Tabla 2.3

Principales hadrones

\begin{tabular}{|c|c|c|c|}
\hline Nombre & Símbolo & Masa $(\mathrm{MeV})^{3}$ & Composición de quarks \\
\hline protón & $\mathrm{p}$ & $938.27231 \pm 0.00028$ & uud \\
\hline neutrón & $\mathrm{n}$ & $939.56563 \pm 0.00028$ & udd \\
\hline sigma + & $\Sigma^{+}$ & $1189.37 \pm 0.07^{4}$ & uus \\
\hline sigma - & $\Sigma^{-}$ & $1197.449 \pm 0.0030$ & $d d s$ \\
\hline
\end{tabular}

\footnotetext{
${ }^{3}$ La información de las masa de las partículas fue tomada del libro "Review of Particle Physics", Volumen 3 Número $1-4,1998$.
} 


\subsubsection{Partículas portadoras}

Uno de los principales cambios en la concepción de los fenómenos entre la física clásica y la física moderna es el concepto de la acción a distancia [14]. En la física clásica la fuerza de gravedad actuaba de forma inmediata entre dos cuerpos. En cambio en la teoría de la relatividad apuntaba a que esto no era así. Existía cierto lapso de tiempo para que la interacción gravitacional entre los dos cuerpos se manifestara. Esto se debe a que existe una velocidad límite en el Universo, y esta es la velocidad de la luz en el vació ${ }^{5}$. Utilizando esto como principio podemos representar un campo para cada una de las fuerza. En Mecánica Quántica podemos representar este campo como una partícula, la cual llamaremos partícula portadora. En condiciones normales estas partículas portadoras no podemos detectarlas. Esto se debe a que el tiempo en el que existe esta dentro de los límites del principio de Heisenberg.

$$
\Delta t \Delta E \leq \hbar / 2
$$

Para poder obsérvalas lo que se hace es aumentar la energía a un nivel en el cual el lapso de tiempo sea lo suficientemente larga para lograr la observación. En la siguiente tabla presentamos la principal información sobre las partículas portadoras.

\section{Tabla 2.4}

Partículas portadoras

\begin{tabular}{|c|c|c|c|c|}
\hline Nombre & Fuerza portadora & Masa & Espín & $\begin{array}{c}\text { Carga } \\
\text { eléctrica }\end{array}$ \\
\hline Fotón & electromagnética & 0 & 0 & 0 \\
\hline $\mathrm{W}^{+}$ & Nuclear débil & $83 \mathrm{GeV} / \mathrm{c}^{2}$ & 1 & +1 \\
\hline $\mathrm{W}^{-}$ & Nuclear débil & $83 \mathrm{GeV} / \mathrm{c}^{2}$ & 1 & -1 \\
\hline $\mathrm{Z}$ & Nuclear débil & $91 \mathrm{GeV} / \mathrm{c}^{2}$ & 1 & 0 \\
\hline gluones & Nuclear fuerte & 0 & 1 & 0 \\
\hline
\end{tabular}

\footnotetext{
${ }^{5}$ En el vació la velocidad de la luz tiene el siguiente valor $\mathrm{c}=2.99792458 \times 10^{8} \mathrm{~m} / \mathrm{s}$ este valor es exacto.
} 


\subsubsection{Leyes de Conservación}

Tanto en la Física Clásica como en la Mecánica Cuántica encontramos que ciertas cantidades físicas se conservan luego de ocurrir un proceso físico. Los ejemplos más notables de este fenómeno es la conservación de la energía y del momentum lineal. En el caso particular de la Mecánica Cuántica encontramos que ciertas cantidades solo aparecen en valores discretos, a esta propiedad le llamamos cuantificación. La gran mayoría de las propiedades de las partículas elementales pueden ser expresadas en términos de estas cantidades discretas, las cuales las llamamos números quánticos. Un ejemplo de esto son los posibles valores del espín de los leptones. Estos valores son $+1 / 2$ o $-1 / 2$. Algo que debemos aclarar es que existen dos clases de conservaciones, el primer grupo son las que se deben a como es el Universo y las otras son la que provienen del modelo que utilizamos para entender el Universo. Al primer grupo pertenecen la conservación de momentum lineal, momentum angular, energía y la conservación de la carga eléctrica.

Tabla 2.5

\section{Leyes de conservación}

\begin{tabular}{|c|c|c|c|}
\hline $\begin{array}{c}\text { Cantidad } \\
\text { Conservada }\end{array}$ & Interacción Fuerte & Interacción Débil & $\begin{array}{c}\text { Interacción } \\
\text { Electromagnética }\end{array}$ \\
\hline Energia/Momentum & $\mathrm{Si}$ & $\mathrm{Si}$ & $\mathrm{Si}$ \\
\hline Carga electrica & $\mathrm{Si}$ & $\mathrm{Si}$ & $\mathrm{Si}$ \\
\hline Número barionico & $\mathrm{Si}$ & $\mathrm{Si}$ & $\mathrm{Si}$ \\
\hline Número leptonico & $\mathrm{Si}$ & $\mathrm{Si}$ & $\mathrm{Si}$ \\
\hline Isoespín & $\mathrm{Si}$ & $\mathrm{No}$ & $\mathrm{No}$ \\
\hline Extrañes & $\mathrm{Si}$ & $\mathrm{No}$ & $\mathrm{Si}$ \\
\hline C (charmess) & $\mathrm{Si}$ & $\mathrm{No}$ & $\mathrm{Si}$ \\
\hline Paridad & $\mathrm{Si}$ & $\mathrm{No}$ & $\mathrm{Si}$ \\
\hline $\mathrm{CP}$ & $\mathrm{Si}$ & $\mathrm{Si}$ & $\mathrm{Si}$ \\
\hline $\mathrm{CPT}$ & $\mathrm{Si}$ & $\mathrm{Si}$ & $\mathrm{Si}$ \\
\hline
\end{tabular}

\subsection{El quark charm : su descubrimiento y características}

A comienzo de la década de los setenta se encontró un resultado interesante una nueva resonancia cuando se realizaba las siguiente colisión [16]. La misma tenía una energía de 
$3.096 \mathrm{GeV}$ y luego otra resonancia fue encontrada a $3.687 \mathrm{GeV}$. La nueva resonancia fue llamada $\psi$ por Burton Richter y su equipo del SLAC (Stanford Linear Accelerator). Esta misma resonancia fue detectada por otro equipo en Brookhaven por el equipo de trabajo de Samuel Ting, quienes lo nombraron J. Por este descubrimiento, ambos recibieron el premio Nóbel en el 1976. Luego la partícula con una masa de $3.097 \mathrm{GeV}$ sería llamada $J / \psi$ y la de masa $3.687 \mathrm{GeV}$ fue llamada J. Los análisis subsiguientes dieron como resultados que estas partículas tenían un nuevo tipo de quark y que las partículas encontradas tenían la misma composición en términos de quarks. La diferencia se debía a que una era resonancia de la otra, en otras palabras un estado excitado. El nuevo quark era el charm. Los mesones descubiertos tenían un charm y un anticharm.

Tabla 2.6

Mesones que contienen charm

\begin{tabular}{|c|c|}
\hline Nombre & Contenido de quarks \\
\hline $\mathrm{D}^{+}$ & $c \bar{d}$ \\
\hline $\mathrm{D}^{-}$ & $\bar{c} d$ \\
\hline $\mathrm{D}^{0}$ & $c \bar{c}$ \\
\hline $\mathrm{D}_{\mathrm{s}}^{+}$ & $c \bar{s}$ \\
\hline
\end{tabular}

\subsection{Breve introducción a la Teoría de la Relatividad}

Las teorías que forman la base de la física de partículas son dos, la Mecánica Cuántica, como hemos mencionando en las pasadas secciones, y la otra es la teoría de la relatividad. Ambas teorías tomaron forma durante las primeras décadas del siglo XX. Un dato curioso es que la Mecánica Cuántica es el producto del trabajo de muchos teóricos y experimentalistas, por otro lado la Teoría de Relatividad [17] no lo es así. La misma tiene un solo autor, el físico alemán Albert Einstien. El publicó su teoría especial en el 1905 y la general en el 1915. La teoría unifica las leyes de la mecánica con el electromagnetismo. Antes del 1905 la naturaleza del movimiento se entendía a través de la idea de Newton de que el tiempo y el espacio eran absolutos. En el electromagnetismo la idea de la existencia de un éter ${ }^{6}$ proveía la base absoluta por la cual se podía determinar el movimiento. La idea de la existencia del éter tuvo un gran revés por el resultado del experimento de Michelson Morley. La interpretación fue que no

6 Fue un invento de Issac Newton y luego reinventado por J.C. Maxwell. Éste llenaba todo el espacio "vacío” del

Universo. La idea era tener un medio por el cual viajaba las ondas electromagnéticas. 
existía el éter. En su teoría Eisntein refutó los conceptos de tiempo y espacio absolutos, su teoría partía de los siguientes dos postulados. Primero, todas las leyes físicas son las mismas para todos los observadores en cualquier marco de referencia inercial. Segundo, la velocidad de la luz es la misma para todos los observadores, independientemente de las velocidades relativas de las fuentes y de los observadores. Eisntein demostró que con estos postulados los requerimientos de equivalencia de lo que veía un observador y otro observador se podían obtener al utilizar las transformaciones de Lorentz. La teoría trajo importantes interpretaciones, las cuales cambiaron todo la forma de ver el Universo.

La transformación del tiempo implica que dos eventos simultáneos de acuerdo a un observador no iban a ser necesariamente igualmente simultáneos para otro observador en movimiento relativamente uniforme. Esto no afecta los conceptos de causalidad. Esto aparece como para observadores en movimiento relativo uniforme sus relojes irán más lentos uno respecto al otro. A este fenómeno se le llama dilatación del tiempo, dada por la siguiente ecuación:

$$
T_{v}=\frac{T_{0}}{\sqrt{1-v^{2} / c^{2}}}
$$

Donde $T_{0}$ es el tiempo del que viaja a la velocidad relativística $T_{v}$ es el tiempo del observador.

En mecánica relativista el momentum y de energía son conservados. Para un observador con velocidad $v$ con respecto a una partícula, la masa de la misma $m$, será determinada por:

$$
m_{v}=\frac{m_{0}}{\sqrt{1-v^{2} / c^{2}}}
$$

Donde $m_{0}$ es la masa en reposo de la partícula. Esta fórmula ha sido experimentalmente comprobada un sinnúmero de veces y es la razón por la cual en los experimentos de física de partículas se aceleran las partículas. Una consecuencia de esto es que cuando la velocidad de una partícula se va acercando a la velocidad de la luz es que la masa 
inercial aumenta lo que hace que la energía requerida para acelerarla se incrementa exponencialmente. Un resultado importante de la teoría es que podemos expresar la energía de cualquier sistema como:

$$
E=m c^{2}(2.4)
$$

El primer intento de expresar la Mecánica Cuántica en términos consistentes con la relatividad fue hecho por Sommerlfeld. Eventualmente Dirac estableció las ecuaciones de ondas mecánicas en términos de la relatividad. Esto sirvió para poder explicar el espín y el momentum magnético asociado. Este trabajo es muy importante para la Física de Partículas, la Teoría del Decaimiento Beta y la Mecánica Estadística. La única limitación que posee el trabajo de Dirac es que el solo se puede aplicar a los fermiones, para el caso de los bosones hay que utilizar la ecuación de Klein-Gordon.

Desde el punto de vista matemático la formulación de la relatividad especial fue dada por Minkowsi. Él se basó en la idea de que un evento se podía especificar por cuatro coordenadas, de las cuales tres son espaciales y la última es temporal. Estas coordenadas definían un espacio cuatro-dimensional y el movimiento de una partícula era representado por una curva en el espacio. A esto se le conoce como el espacio de Minkowski.

En nuestro caso para estudiar los decaimientos la teoría de la relatividad nos es útil para poder determinar el tiempo propio, el cual es dado por la siguiente ecuación:

$$
\tau=\left(\frac{L}{c}\right)\left(\frac{m}{p}\right)
$$

Donde $\tau$ es el tiempo propio. Este tiempo es definido como el intervalo entre dos eventos que son medidos por un observador. Quien determina que los eventos ocurren en el mismo punto en el espacio. En nuestro caso $L$ es la distancia entre el vértice de producción y el vértice de decaimiento, $c$ es la velocidad de la luz, $m$ es la masa de la partícula y $p$ es su momentum.

La teoría especial de la relatividad solo puede ser utilizada para los casos en los cuales el observador tiene un movimiento relativo a un marco de referencia en reposo. Para los casos donde el observador y el observado están en movimiento se tiene que utilizar la teoría 
general.

\subsection{Experimentos antes de FOCUS}

Desde el descubrimiento del quark charm en la década de los sesenta, se han realizado varios experimentos para estudiarlo. Los experimentos WA75 [18] y E653 ${ }^{7}$ [19] fueron dos experimentos en los cuales se estudiaron partículas que contenían charm. Se utilizaba placas fotográficas especiales en las cuales quedaban marcadas las trayectorias, luego con el uso de un microscopio se reconstruían las trayectorias. Esto daba una alta resolución de reconstrucción de trayectorias pero una baja estadística por lo complicado y costoso del método. Al pasar el tiempo y mejorar la tecnología se fue haciendo más fácil la construcción de mejores detectores haciendo posible experimentos más complejos.

Uno de los principales experimentos en la historia de los estudios realizados sobre el quark charm fue el E691 [20] realizado en Fermilab. En este experimento se reconstruyeron unas 10,000 trayectorias que contenían charm. Para hacer la reconstrucción de las trayectorias se hizo una gran innovación que fue la utilización de detectores de silicio cerca del detector. Esta innovación dio como resultado una muy buena resolución de las partículas que contenían charm. Estas imposible de reconstruir con otros detectores por la distancia que pueden viajar antes de decaer.

Otros experimentos en los cuales se estudió el quark charm fueron CLEO y E-791 [21]. Los mismos dieron una alta estadística pero no una alta resolución ya que las trayectorias de las partículas que contenían charm no eran reconstruidas. Además, en el caso de CLEO el mecanismo de producción era algo diferente a los demás, el mismo consistía en colisionar un electrón y positrón. En física de partículas existen dos formas principales de hacer los experimentos, los de blanco fijo y los de colisión.

En el caso de E791 el método de producción que se utilizó fue producción hadrónica. El mismo consiste en disparar un haz de hadrones contra un blanco fijo, el haz consistía de piones. La configuración de los detectores fue parecida en términos de la posición y tipos utilizados. Una de las dificultades utilizando es que la producción hadrónica aunque produce más partículas con charm que la fotoproducción, la principal fuente de ruido se debe a la misma fuente lo cual hace muy difícil su filtración. En cambio la fotoproducción la mayor fuente

7 En Fermilab se utiliza la notación E\#\#\# para designar los experimentos. 
es de origen electromagnético mediante la producción de pares conjugado de electrón-positrón.

\subsection{Descripción de FOCUS}

\subsubsection{Objetivos de FOCUS}

Los objetivos de FOCUS eran los siguientes:

1. Estudios precisos de decaimientos semileptónicas.

2. Análisis detallados de las gráficas de Dalitz para varios canales decaimiento de mesones de charm.

3. Estudio de QCD utilizando pares conjugados de charm.

4. Una medición de fracciones absolutas de razones de decaimiento para el mesón $D^{0}$.

5. Espectroscopia de los estados excitados de mesones con charm.

6. Estudios de varios decaimientos doblemente suprimidos por Cabibbo.

7. Una investigación sistemática de las partículas que contengan charm de las masas de los bariones, vidas medias, anchos de las señales, y branching fractions.

8. Búsqueda de $D^{0}$ "mixing", violación de $\mathrm{CP}$, decaimientos raros o prohibidos.

9. Reconstrucción de los decaimientos semileptónicos de la partícula $\mathrm{D}+$.

\subsubsection{Descripción General}

El experimento FOCUS ("Fotoproduction of Charm with Upgraded Spectometer") $[22,23,24,25,26]$ fue un experimento realizado del 1995 al 1998. Fue un experimento de blanco fijo, que utilizó el método de fotoproducción, esto era un rayo de fotones de alta energía disparados contra un blanco fijo de óxido de berilio. Durante el período que se corrió el experimento se colectaron 5,925 cintas magnéticas de $4.4 \mathrm{~Gb}$ de datos en cada una de ellas, para un total aproximadamente $23 \mathrm{~Tb}$. En éstas estaban la información de más de un millón de eventos que contenían partículas con charm. En estos eventos había decaimientos de mesones y bariones. La cantidad de datos obtenidos convierte a FOCUS en el mejor experimento de su tipo realizado hasta ese momento en base a la cantidad de datos obtenidos.

En esencia los detectores del experimento forman un espectrómetro de apertura ancha de masa de múltiple partículas. Esto quiere decir que se puede detectar muchas partículas 
de diferentes masas con los mismos detectores. La razón para esto es porque las partículas que contienen charm al decaer las partículas hijas lo hacen en partículas que tienen una gama bien variada de masas. El espectrómetro esta dividido en dos partes las cuales se denominan como "inner" y "outer". La sección "outer" es la que está diseñada para detectar partículas cuyas trayectorias tienen un ángulo pequeño con respecto al eje longitudinal del espectrómetro. Esta sección es la que queda antes del segundo magneto. Por otra parte la sección posterior al segundo magneto se conoce como "inner", la misma está diseñada para detectar las partículas con ángulos mayores con respecto al eje central o longitudinal. Un detalle importante es que el espectrómetro posee dos magnetos. Es conveniente que el lector observe la figura 2.1 para que tenga una mejor idea de la configuración general del espectrómetro.

En términos generales los detectores se puede dividir en dos grupos principales, los detectores de posición y de identificación. Al primer grupo pertenece los $\mathrm{PWC}^{8}$, el detector de silicio del blanco o TSD y los "Silicon Micro Strips". Estos detectores nos sirven para poder reconstruir las trayectorias de las partículas cargadas. Utilizando la información de todos estos detectores es que podemos calcular las trayectorias de las partículas con carga eléctrica. Cuando una partícula con carga eléctrica pasa por el área produce

De todos los detectores de posición el más importante para nuestro trabajo es el TSD. Para la descripción del mismo dedicamos una sección completa (sección 2.5.5). Luego del área del blanco se encuentra el SSD. El mismo está compuesto de 12 planos agrupados en 4 estaciones de 3 planos cada una. La orientación de estos planos está diseñada para que cada uno de ellos midiera una de las coordenadas $i, j, \mathrm{y} k$. La orientación de cada uno de los planos es las siguiente, para el plano $i$ está a $-135^{\circ}$, el plano $j$ está a $-45^{\circ}$ y finalmente el plano $k$ a está a $-90^{\circ}$; todos orientados con respecto a la dirección del eje de $x$ del marco de coordenadas escogido para el experimento. En la siguiente figura nos muestras dónde está cada una de las estaciones del SSD y las distancias relativas al área o región del blanco.

${ }^{8} \mathrm{PWC}$ es el acronimo en ingles para "Propotional Wire Chamber" 


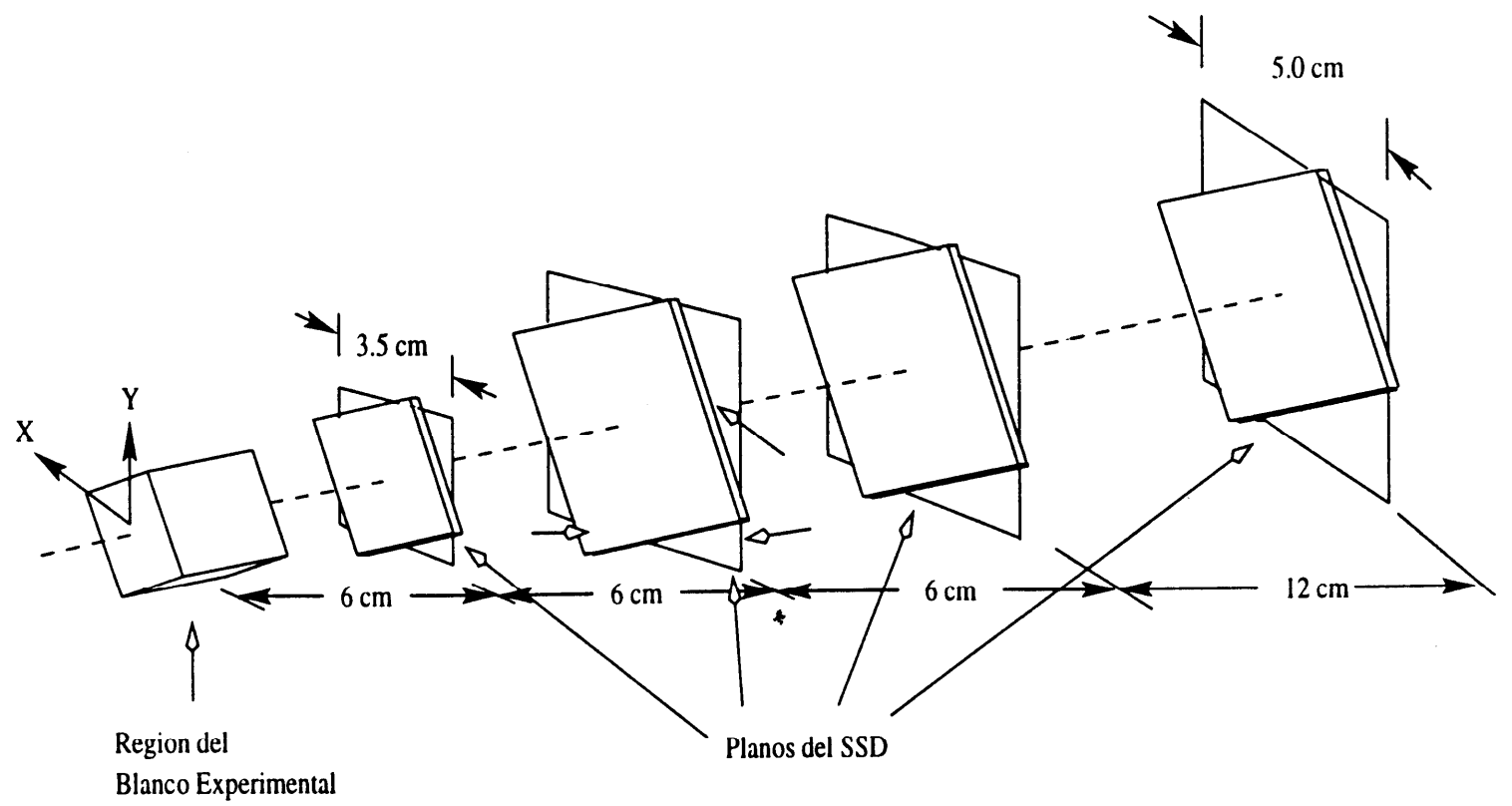

Figura 2.1

Configuración del SSD

Cada uno de los detectores fue montado en unos soportes construidos de granito con una alta precisión, lo cual nos aseguró alineamiento del sistema de unos $\pm 3 \mu \mathrm{m} / 5 \mathrm{~cm}$. La razón para tener una precisión tan alta era que estos detectores nos proveen la principal información para la reconstrucción de las trayectorias. Esta información es importante para determinar el momentum de estas partículas y con esto poder calcular el momentum de la partícula madre o que contiene charm. En la siguiente tabla desglosamos las principales características del detector. 
Tabla 2.7

Principales características del SSD

\begin{tabular}{|c|c|c|c|c|}
\hline Detector & Estación 1 & Estación 2 & Estación 3 & Estación 4 \\
\hline $\begin{array}{c}\text { Primer plano } \\
\text { Segundo } \\
\text { plano }\end{array}$ & 0.5 & 5.5 & 11.5 & 23.5 \\
\hline $\begin{array}{c}\text { Tercer plano } \\
\text { Área activa } \\
\left(\mathrm{cm}^{2}\right)\end{array}$ & 0.5 & 6 & 12 & 24 \\
\hline $\begin{array}{c}\text { Área de alta } \\
\text { resolución } \\
\left(\mathrm{cm}^{2}\right)\end{array}$ & 3.5 & 6.5 & 12.5 & 24.5 \\
\hline $\begin{array}{c}\text { Esparcimiento } \\
(\mu m)\end{array}$ & $25 / 50$ & $50 / 100$ & $50 / 100$ & $50 / 100$ \\
\hline $\begin{array}{c}\text { Número de } \\
\text { canales }\end{array}$ & 688 & 688 & 688 & 688 \\
\hline
\end{tabular}

En el mismo grupo de detectores de posición se encuentra los PWC. Estos se encontraban luego del primer magneto. Estaba agrupada en 5 estaciones cada una conteniendo 4 planos, para formar un total de 20 planos. Las estaciones estaban identificadas por P0, P1, P2, P3 y P4. En ese orden era el que se encontraban a lo largo del eje de z. El primer grupo de estaciones estaba comprendido por P0, P1 y P2, éstas se encontraban entre los dos magnetos. P0 se encontraba justamente luego del primer magneto. En cambio las estaciones P1 y P2 estaban luego de los detectores de Čerenkov. Las estaciones restantes de los PWC se encontraban luego del segundo magneto. P3 estaba justamente luego del segundo magneto y P4 estaba luego del último detector de Čerenkov. La orientación de los planos en cada una de las estaciones fue la misma. Existían cuatro orientaciones, X, Y, U, y V. Las orientaciones X y Y eran en la mimas dirección de los ejes de las coordenadas del experimento. En cambio U estaba orientado $+11.3^{\circ}$ con respecto al eje de $\mathrm{Y}$, por otro lado el plano $\mathrm{V}$ lo estaba $-11.3^{\circ}$ con respecto al mismo plano. La disposición de los plano en cada una de las estaciones fue la siguiente: Y, V, U, y X. La estaciones se pueden dividir en dos grupos, denominadas como Tipo I y Tipo II. La que son Tipo I tienen una apertura de $0.762 \mathrm{~m}$ por $1.27 \mathrm{~m}$ y funcionaban en un rango de voltaje de $3.30 \mathrm{kV}$ a $3.50 \mathrm{kV}$. La estaciones Tipo II tenían una apertura de $1.52 \mathrm{~m}$ por $2.28 \mathrm{~m}$ y fusionaban en un rango de voltaje de $3.00 \mathrm{kV}$ a $3.20 \mathrm{kV}$. Los rangos de voltajes fueron obtenidos durante el período de 
calibración que se realizó durante un año aproximadamente antes de la corrida experimental. En la siguiente figura presentamos la orientación de los diferentes planos de los PWC.

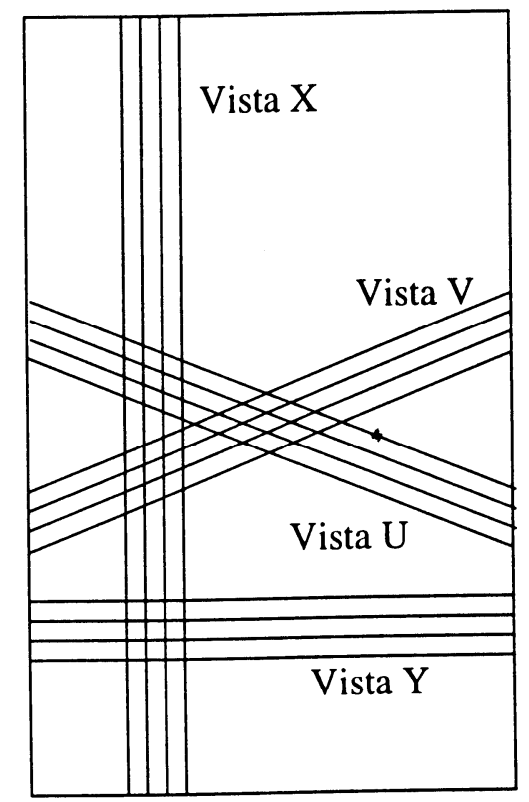

Figura 2.2

Configuración de los planos del PWC

El siguiente grupo de detectores son para la identificación. A este grupo pertenecen de los detectores de Čerenkov [27], y la además los calorímetros hadrónicos y electromagnético. Los detectores de Čerenkov se utilizan para identificar las partículas con carga y los calorímetros principalmente son utilizados para detectar las partículas neutrales, aunque también las partículas con carga pueden dejar señal. Para no utilizar esta señal se tiene una serie de detectores auxiliares los cuales se le llaman gatillos. El propósito de este es aceptar o rechazar trayectorias en la reconstrucción de eventos.

Los detectores de Čerenkov de nuestro experimento fueron todos del tipo umbral $[28,29]$. Un detector Čerenkov funciona de la siguiente manera. Cuando una partícula viaja a una velocidad que es más rápida que la velocidad de la luz en ese medio entonces la partícula emite fotones. Estos fotones forman un frente de onda, algo parecido en la geometría a cuando una aeronave rompe la barrera del sonido. Hay que recordar que la velocidad de la luz en el medio es menor que en el vacío y por consiguiente no se está violando la Teoría de la Relatividad. La 
condición para que ocurra este fenómeno está resumida en la siguiente ecuación:

$$
\beta=\left(\frac{v}{c}\right)=\frac{v_{u m b r a l}}{c}=\frac{1}{n}(2.6)
$$

Donde $n$ es el índice de refracción del medio $v$ es la velocidad de la partícula. En la siguiente figura representamos la geometría de la emisión de luz Čerenkov.

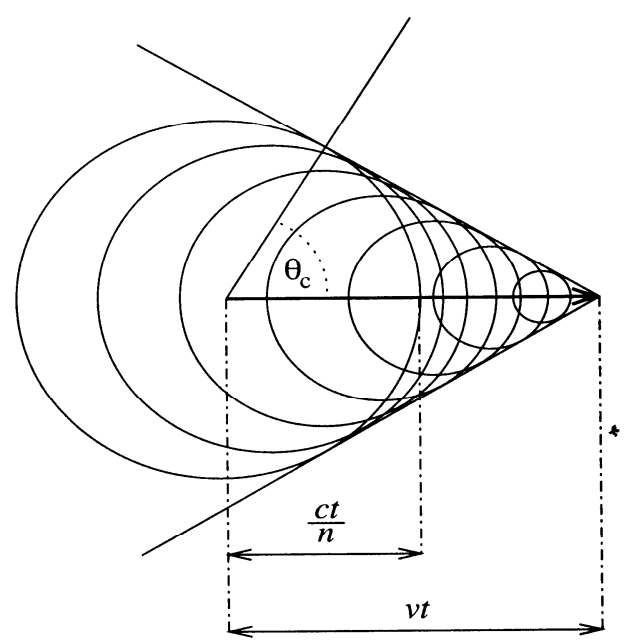

Figura 2.3

Emisión de la producción de luz Čerenkov ${ }^{9}$

El ángulo en que se emite la radiación de Čerenkov está inversamente relacionado con la velocidad de la partícula y con el índice de refracción del medio y está dada por la siguiente relación:

$$
\cos \theta=\frac{1}{\beta n}(2.7)
$$

Es esta ecuación podemos determinar cuál es el ángulo máximo de emisión, el cual en el caso de que $\theta=1$ será:

$$
\cos \theta_{\text {sat }}=\frac{1}{n}(2.8)
$$

9 Esta imagen fue tomada del libro de J. Jackson titulado: Classical Electrodynamics. 
Debemos recordar que cuando $\theta=1$ es cuando $\beta=c$. Para determinar el número promedio de fotones emitidos en el proceso por unidad de longitud tenemos que esto es dado por:

$$
\frac{d N}{d x} \propto C \sin ^{2} \theta(2.9)
$$

Donde $\theta$ es el ángulo de Čerenkov. El número promedio de los fotones emitidos será proporcional a $\sin ^{2} \theta$ ya que el espectro de fotones es independiente de $\theta$. Si observamos una gráfica donde sea el momentum de la partícula versus el número de fotones emitidos veríamos los siguiente.

$$
\begin{gathered}
\gamma_{\text {umbral }}=\frac{1}{\sqrt{1-\beta_{\text {umbral }}^{2}}}=\frac{n}{\sqrt{n^{2}-1}} \\
p_{x}=\gamma_{\text {umbral }} \nu_{\text {umbral }} m_{x}
\end{gathered}
$$

Donde $p_{x}$ es el umbral de momentum de la partícula $x$. La pasada expresión que es útil para nuestros propósitos es la razón de los umbrales de diferentes partículas que surgen con una consecuencia de la ecuación penúltima. Se puede calcular el umbral de momentum de una partícula conociendo el umbral de momentum de otra para un medio determinado y adicional teniendo la razón de las masas correspondientes. Un ejemplo seria la siguiente ecuación es una comparación entre la masa del kaón y del pión.

$$
p_{\text {umbral } \pi}=p_{\text {umbralK }} \frac{m_{K}}{m_{\pi}}
$$

Los detectores de Čerenkov de nuestro experimento eran de umbral. Esto quiere decir que solo se puede saber si se emitió la luz. Por esta razón se utilizaron en nuestro experimento tres detectores. Esto nos permitía determinar si era una partícula o otra dependiendo de cuales fueron los que se 
emitieron luz. En el caso de los Čerenkov en nuestro espectrómetro teníamos 3 de ellos. Los primeros dos $\mathrm{C} 1$ y $\mathrm{C} 2$ estaban localizados entre los dos magnetos y C3 luego del segundo magneto.

El primero de los detectores $\mathrm{C} 1$, estuvo luego del primer magneto y entre dos estaciones del PWC. En el mismo se utilizo una mezcla de helio y nitrógeno a presión atmosférica. La longitud interior del mismo era de $1.803 \mathrm{~m}$. En su interior tenía un total de 90 celdas cada una de las cuales apuntaban a un tubo foto multiplicador. Los espejos estaban orientados a 45 grados con respectos al eje longitunidal. En la siguiente figura está la vista superior del detector.

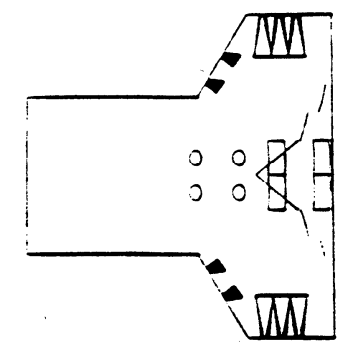

Figura 2.4

Detector C1, vista superior

El siguiente detector que encontramos en la dirección que viaja el rayo de fotones es el C2. Éste estaba localizado entre P1 y P2, y tenía una apertura de 3.71 metros cuadrados (Siendo $1.625 \mathrm{~m}$ de ancho y $2.286 \mathrm{~m}$ de altura). En contraste con el detector anterior tenía dos grupos de celdas; las interiores que eran unas 54 y las grandes que eran unas 56 que se situaban en la periferia, sumando a un total de 110 celdas. El gas que se utilizo fue $\mathrm{N}_{2} \mathrm{O}_{\text {a presión }}$ atmosférica.

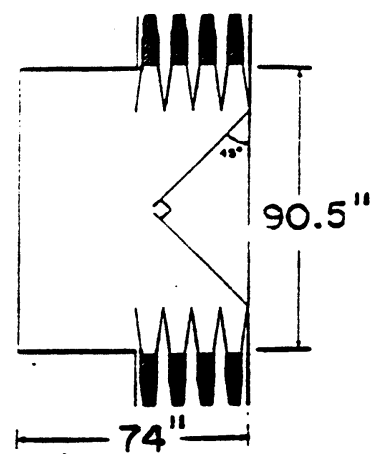

Figura 2.5

Detector C2, vista superior 
El último de los detectores de Čerenkov, se encontró luego del segundo magneto y después del Sistema Exterior de Muones, entre la estación P3 y P4 de los PWC. El gas utilizado esta vez fue $H e$ y también estaba a presión atmosférica.

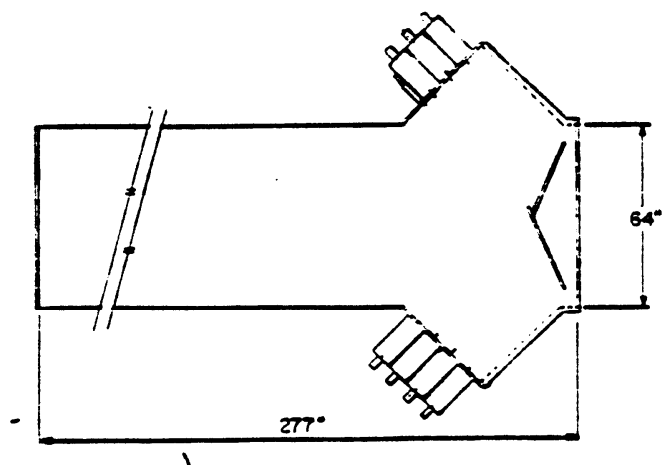

Figura 2.6

Detector C3, vista superior

En la siguiente tabla presentamos las principales características de los tres detectores de Čerenkov.

Tabla 2.8

Características principales de los Čerenkov

\begin{tabular}{|c|c|c|c|c|c|c|}
\hline Detector & $\begin{array}{c}\text { Número } \\
\text { de } \\
\text { células }\end{array}$ & $\begin{array}{c}\text { Largo } \\
(\mathrm{cm})\end{array}$ & $\begin{array}{c}\text { Gases } \\
\text { Utilizados }\end{array}$ & $\begin{array}{c}\text { Umbral } \\
\text { del pión } \\
(\mathrm{GeV})\end{array}$ & $\begin{array}{c}\text { Umbral } \\
\text { del kaón } \\
(\mathrm{GeV})\end{array}$ & $\begin{array}{c}\text { Umbral } \\
\text { del } \\
\text { protón } \\
(\mathrm{GeV})\end{array}$ \\
\hline $\mathrm{C} 1$ & 90 & 188 & $\mathrm{He}-\mathrm{N}$ & 8.4 & 29.8 & 56.5 \\
\hline $\mathrm{C} 2$ & 110 & 188 & $\mathrm{~N}_{2} \mathrm{O}$ & 4.5 & 16 & 30.9 \\
\hline $\mathrm{C} 3$ & 100 & 711 & $\mathrm{He}$ & 17.4 & 61.8 & 117 \\
\hline
\end{tabular}


Para cada trayectoria detectada por los PWC, el algoritmo compara el patrón de luz que se espera si $p>p_{\text {umbral }}$ con el patrón observado. Existe un $p_{\text {umbral }}$ diferente para cada tipo de partícula en cada uno de los detectores de Čerenkov. Se calcula el número de fotones esperado y se calcula el tamaño del cono de luz. Luego se compara el número de fotones esperados con el observado en la celda principal y en las celdas adyacentes. Si la señal es consistente con ese número de fotones (o mayor) se dice que la información del Čerenkov es consistente con que la trayectoria sea de ese tipo de partícula. Para lograr la identificación se creo un código que es basado utilizando la señal de cada uno de los detectores como una expresión lógica.

Tabla 2.9

Códigos de Istapt

\begin{tabular}{|c|c|}
\hline Código de istapt & Interpretación \\
\hline 0 & inconsistente \\
\hline 1 & electrón \\
\hline 2 & pion \\
\hline 3 & Electrón, pion \\
\hline 4 & kaon \\
\hline 6 & Pion, kaon \\
\hline 8 & protón \\
\hline 9 & Protón, electrón \\
\hline 10 & Pion, protón \\
\hline 12 & Kaon, protón \\
\hline 15 & inconsistente \\
\hline & \\
\hline
\end{tabular}

En el caso de los calorímetros son lo que se podría llamar detectores destructivos. Esto se debe a que para realizar la detección de una partícula lo que hace es provocar que esta interactuara con el material pasivo del detector y miden la energía depositada en ellos. En nuestro experimento exciten dos tipos de calorímetros los electromagnéticos y los hadrónicos. Otro grupo 
de detectores importantes son los gatillos. Éstos utilizan para seleccionar un evento o rechazarlo.

Un detalle del espectrómetro es que la configuración general del mismo es parecida a la de E687. Los principales cambios fueron:

1.Se aumentó la resolución en el tiempo.

2.Se aumentó la capacidad de procesar datos.

3.Se incrementó la sensitividad de algunos detectores.

A continuación esta una figura del espectrómetro

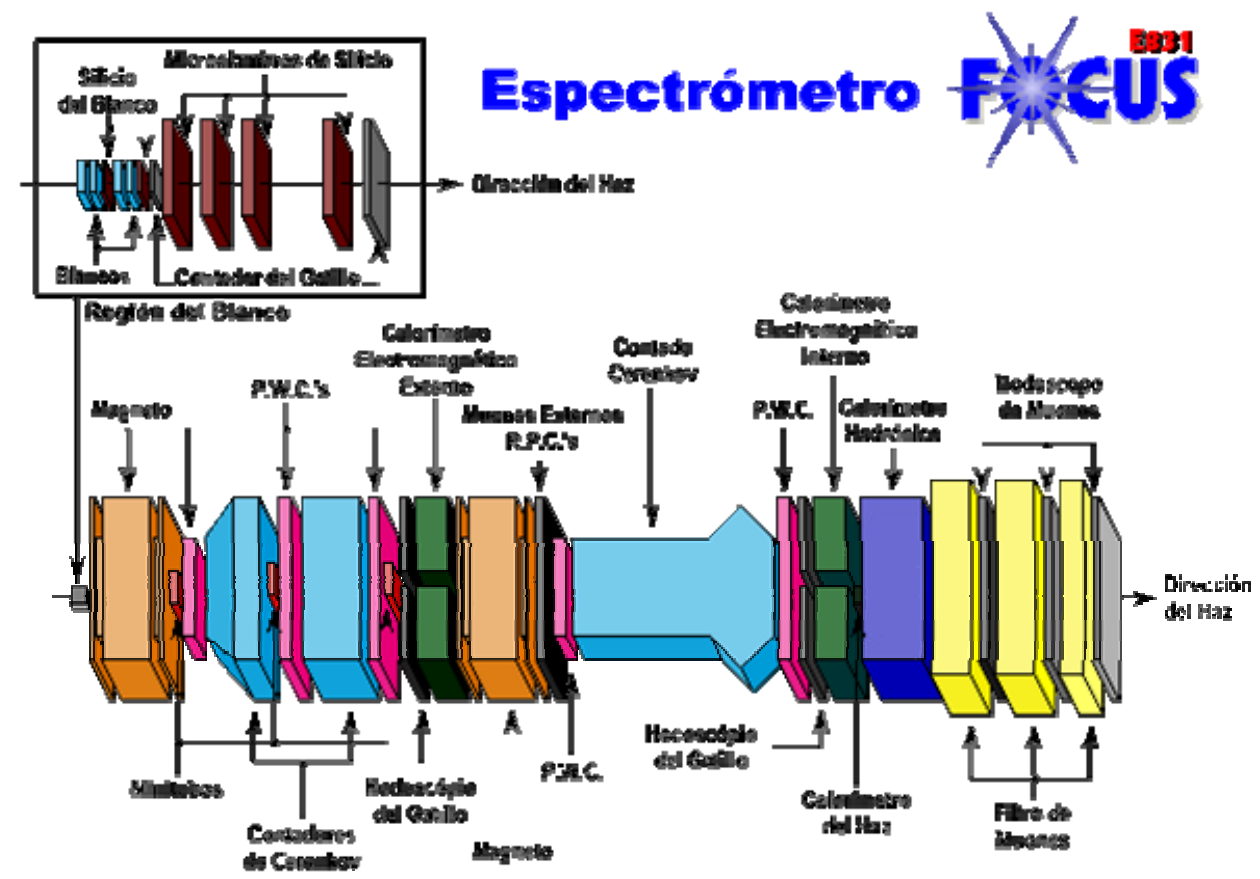

Figura 2.7

Vista general del espectrómetro

\subsubsection{Fotoproducción}

En los experimentos de blanco fijos existen dos métodos de producción, fotoproducción [24] y producción hadrónica [25]. En el caso de la fotoproducción es que un rayo de fotones de alta energía se dispara contra el blanco experimental. Por otra parte en el caso de producción hadrónica se dispara un rayo de hadrónes, este pueden ser de piones, de kaones o más simple de protones. Se intenta siempre utilizar algún hadrón que tenga una vida media bastante larga como para que la partícula se pueda ser útil para los propósitos experimentales, en otras 
palabras que se puede acelerar lo suficiente para alcanzar un momentum suficientemente alto para que la interacción deseada ocurra.

La fotoproducción tiene las siguientes características que la hacen útil para nuestro experimento. La gran mayoría del ruido es de origen electromagnético, por la producción de pares de conjugados de electrones-positrones. Pueden ser fácilmente eliminados haciendo un arreglo en la configuración de nuestros detectores. La gran mayoría del ruido de origen hadrónico proviene de partículas neutras que son producidas en los núcleos. Este luego es relativamente fácil de eliminar mediante las partículas que se producen mayormente son $\pi^{0} \mathrm{y} \mathrm{K}^{0}$, las cuales tiene una vida media relativamente larga y esto tiene la consecuencia que al decaer está algo lejos del vértice primario. Otra cosa es que el $0.6 \%$ de los eventos en la fotoproducción tienen hadrónes en el estado final versus $0.08 \%$ en el caso de la producción hadrónica. Entre sus ventajas tenemos que los eventos son muy limpios, tan limpios que se debe tener detectores muy cerca del blanco para poder detectar el vértice primario. Otro de los problemas es que se requiere un blanco relativamente anchos y esto incrementa la dispersión, lo cual afecta la reconstrucción del vértice secundario. Por esta razón en el experimento FOCUS se utilizó un blanco segmentado. Esto fue un resultado empírico que se obtuvo de E687. Al estar el blanco segmentado los vértices secundarios estaban en su mayoría a fuera. Esto facilitaba la reconstrucción del vértice. Al segmentar el blanco experimental este espacio se pudo utilizar para poner nuevos detectores lo cual mejoraba la reconstrucción del vértice primario.

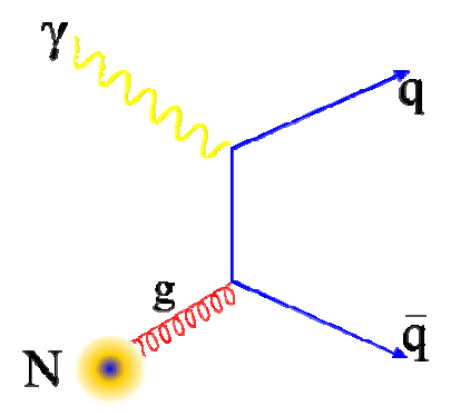

Figura 2.8

Diagrama de la Foto producción

Un factor importante es que el rayo de fotones tiene que ser lo más limpio que se pueda. Si el mismo está contaminado con otras partículas, se afecta de una forma considerable la 
calidad de la producción. Esto nos lleva a tener que utilizar técnicas algo especiales para producir el rayo de fotones.

El experimento de FOCUS requería un haz de fotones que tuviera las siguientes características. Primero tenía que tener una alta energía, $180 \mathrm{GeV}$ en promedio. Segundo el haz tenía que ser lo más limpio posible. Para producir el haz de fotones se utilizaron protones que provenían del Tevatrón. Al momento de realizarse FOCUS el Tevatrón era el acelerador de partículas más potente del mundo. En la actualidad en el laboratorio europeo de CERN está en construcción el LHC el cual ocupara la posición de la que goza el Tevatrón en la actualidad.

El proceso de obtención de los protones necesarios para luego producir el haz de fotones está dividido en varias partes y consta de ciclos que tienen una duración de un minuto. En un principio se tiene hidrógeno, como sabemos el principal isótopo solo consta de un protón en el núcleo y un electrón. Para poder ser acelerado tiene que tener carga eléctrica, por esta razón se le añade un electrón. Se aceleran para luego ser colisionados con un blanco de carbón. Al ocurrir la colisión los electrones se desprenden del núcleo. Luego los electrones y los protones se separan mediante un campo magnético. Los electrones descartados y los protones son dirigidos para ser utilizados en la siguiente etapa. Los protones son dirigidos al Main Ring y luego al Tevatrón, aquí el proceso tiene ciclos de una duración de un minuto. En los primeros 40 segundos se aceleran los protones y en los 20 segundos siguientes se extraen hacia el blanco experimental. El periodo de extracción se conoce como "Spill" o derrame. Los protones que salen en el derrame se agrupan en lo que se denomina como "buckets". Estos tienen un largo de aproximadamente 1 ns y en un espacio entres cada uno de $18.83 \mathrm{~ns}$.

Cada uno de los paquetes o "Buckets" contienen entre $3 \times 10^{12} \mathrm{a}$ $12 \times 10^{12}$ protones. Posteriormente los protones se extraen lentamente y se dividen en tres, los diferentes experimentos de blanco fijo. El número promedio de protones disponibles para el experimento de FOCUS fue de unos $4 \times 10^{12}$.

Para el experimento de FOCUS se necesitaba un haz de fotones que estuviera lo más limpio posible. Este haz se obtenía luego de varios procesos. En el primero el haz de protones colisiona con un blanco de deuterio líquido. Los productos de este proceso son fotones y varias partículas neutras, como piones neutrales. Un poco más adelante los fotones son convertidos a pares de conjugados de electrones. Ahora es fácil de separar los electrones de las demás partículas neutrales que se produjeron anteriormente. Los electrones se separan mediante la utilización de electroimanes y las partículas neutras son absorbidas por un filtro. 
Luego los electrones y positrones son separados mediante la utilización de campos magnéticos, para ser posteriormente redirigidos al radiador del experimento. Ocurre un hecho importante, es que en varios buckets solo se tiene un electrón o un positrón o no se tiene nada. Al estos interactuar con el radiados producen uno o más fotones. Luego del radiador se encuentra otro electro magneto para separar los electrones que no interactuaron con el radiador de los fotones. Los fotones se dirigen al blanco experimental y los electrones se dirigen al RESH y los positrones de dirigen al POSH. Utilizando los datos de estos dos detectores se puede estimar la energía del rayo de fotones. En el siguiente diagrama está representado gran parte de todo el proceso que acabamos de explicar.

\section{Haz de Fotones de Doble Banda}

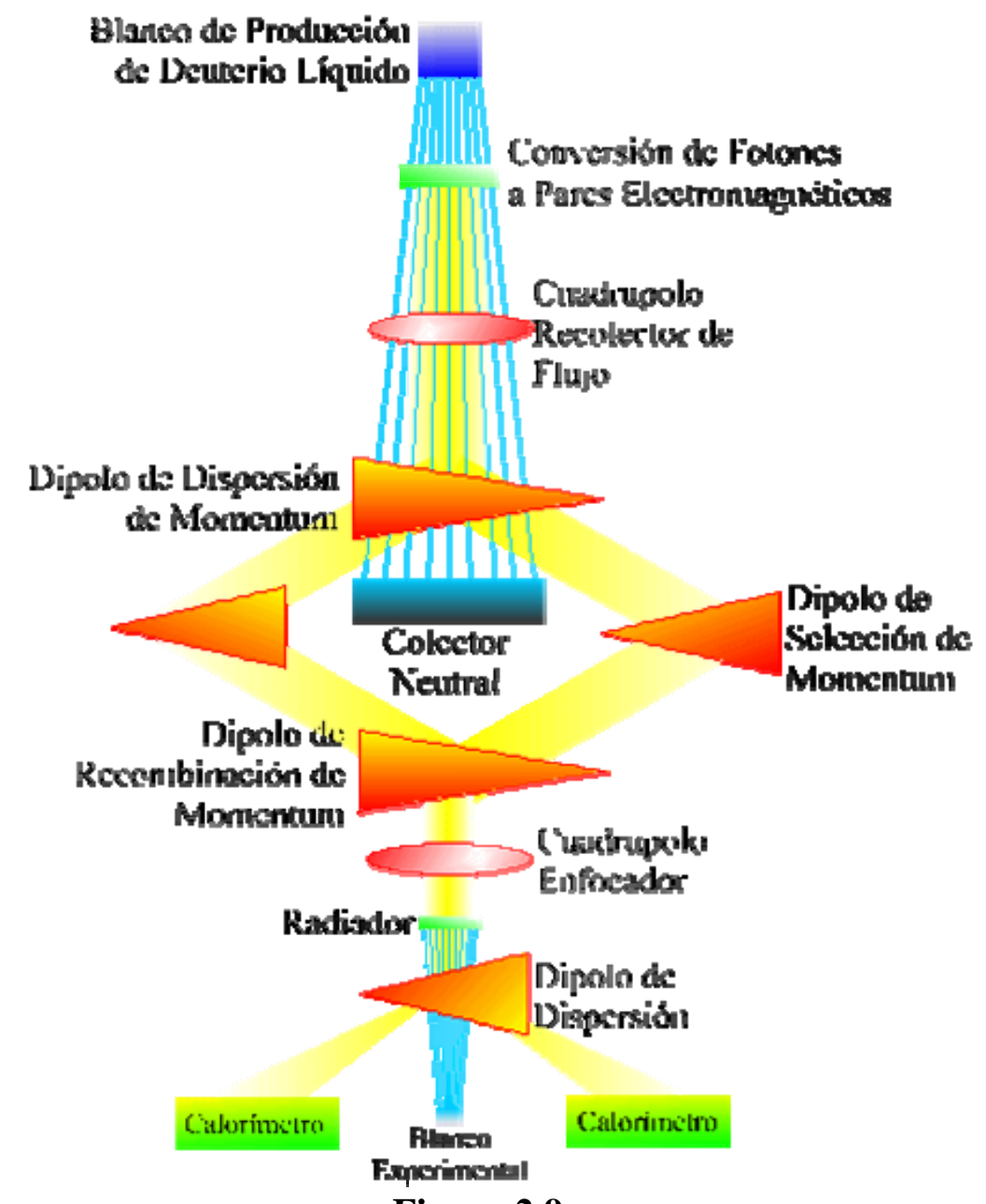

Figura 2.9

Producción del rayo de fotones

En la siguiente tabla tenemos la información referente a las diferentes etapas de la producción del rayo de fotones en las etapas previas al "Swithyard". Presentamos las energías en 
las diferentes etapas.

Tabla 2.10

Descripción de las etapas de la producción del rayo de fotones

\begin{tabular}{|c|c|c|c|}
\hline Acelerador & $\begin{array}{c}\text { Energía inicial del } \\
\text { rayo }\end{array}$ & $\begin{array}{c}\text { Energía final del } \\
\text { rayo }\end{array}$ & Destino del rayo \\
\hline Pre Acelerador & $0 \mathrm{KeV}$ & $750 \mathrm{KeV}$ & Linac \\
\hline Linac & $750 \mathrm{KeV}$ & $400 \mathrm{MeV}$ & Booster \\
\hline Booster & $400 \mathrm{MeV}$ & $8 \mathrm{GeV}$ & Main Ring \\
\hline \multirow[t]{2}{*}{ Main Ring } & $8 \mathrm{GeV}$ & $\begin{array}{c}120 \mathrm{GeV} \text { para la } \\
\text { producción de } \\
\text { antiprotones }\end{array}$ & $\begin{array}{l}\text { Zona de } \\
\text { producción de } \\
\text { antiprotones }\end{array}$ \\
\hline & & $\begin{array}{c}150 \mathrm{GeV} \text { para la } \\
\text { inyección del } \\
\text { Tevatrón }\end{array}$ & Tevatrón \\
\hline \multirow[t]{2}{*}{ Tevatrón } & $150 \mathrm{GeV}$ & $\begin{array}{c}800 \mathrm{GeV} \text { para el } \\
\text { switchyard }\end{array}$ & switchyard \\
\hline & & $\begin{array}{c}900-1000 \mathrm{GeV} \\
\text { para el } \\
\text { colisionador }\end{array}$ & $\begin{array}{c}\text { Experimentos de } \\
\text { colisiones }\end{array}$ \\
\hline $\begin{array}{c}\text { Fuente de } \\
\text { antiprotones }\end{array}$ & $8 \mathrm{GeV}$ & $\begin{array}{c}8 \mathrm{GeV} \text { para el } \\
\text { colisionador }\end{array}$ & $\begin{array}{c}\text { Experimentos de } \\
\text { colisiones }\end{array}$ \\
\hline Switchyard & $800 \mathrm{GeV}$ & $800 \mathrm{GeV}$ & $\begin{array}{c}\text { Experimentos de } \\
\text { blanco fijo }\end{array}$ \\
\hline
\end{tabular}




\subsubsection{Blanco de Óxido de Belirio}

El blanco que se utilizó en FOCUS fue uno de oxido de berilio [30]. Se escogió este material porque su densidad es relativamente alta, $2.97 \mathrm{gm} / \mathrm{cm}^{3}$, esto nos provee un blanco compacto, y un número bajo de electrones. El propósito de tener un número bajo de electrones es para de esta forma reducir la producción de pares conjugados. El blanco era segmentado, no era un blanco único como en el experimento E687. Uno de los descubrimientos empíricos, fue que era mejor para la reconstrucción de los eventos tener el vértice de decaimiento fuera del material del blanco. De esta forma se evitaba que se tuvieran otras interacciones. En nuestro caso el blanco se segmentó en cuatro pedazos. Además el espacio entre los blancos se aprovechó para colocar los detectores de silicio del blanco.

Cada uno de los blancos consistía de un segmento de $3.0 \mathrm{~cm}$. cuadrados y de $6.74 \mathrm{~cm}$. de largo. El espacio entre cada uno de los blancos fue de $1.0 \mathrm{~cm}$. La configuración del área del blanco, que es como nombraremos esta sección del espectrómetro, cambió de configuración durante diferentes períodos del experimento. Se cambió 6 veces de configuración debido a que se fue perfeccionando el experimento mientras se iba corriendo. En la siguiente figura se tiene una vista superior del área del blanco.

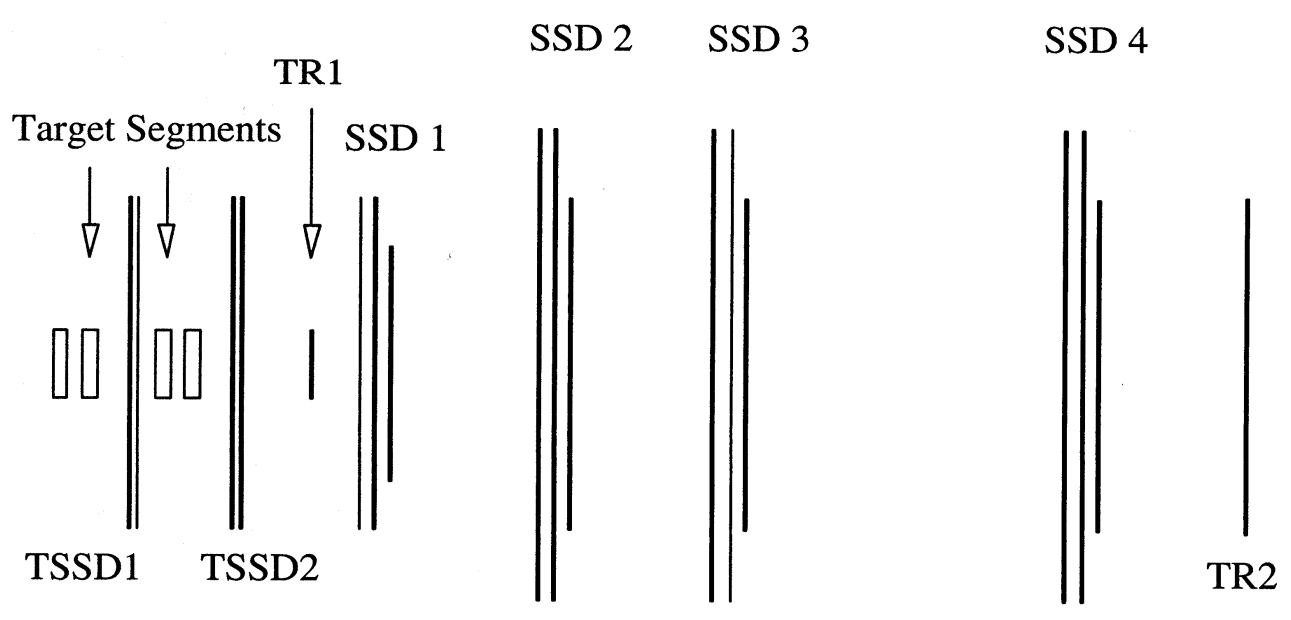

FIGURA 2.10

Región del blanco. 
El tiempo en que se corrió el experimento está dividido en períodos. La diferencia entre cada uno de ellos mayormente es la configuración de alguna parte del espectrómetro. Al principio el experimento se empezó a correr con el blanco que fue utilizado en E-687. Los principales cambios entre período y período radican en:

a- La configuración del blanco experimental.

b- La posición de algunos de los detectores.

c- La introducción de detectores, como fue el caso del TSD.

En la siguiente tabla presentamos los datos correspondientes al área del blanco para los diferentes períodos con la información de la posición de los diferentes segmentos del blanco.

Tabla 2.11

Datos de la geometría del área del blanco por período

\begin{tabular}{|c|c|c|c|c|}
\hline Periodo & Plano I & $\begin{array}{c}\text { Plano II } \\
(\mathrm{cm})\end{array}$ & $\begin{array}{c}\text { Plano III } \\
(\mathrm{cm})\end{array}$ & $\begin{array}{c}\text { Plano IV } \\
(\mathrm{cm})\end{array}$ \\
\hline I & 5.1 a 0.7 & $\mathrm{n} / \mathrm{a}$ & $\mathrm{n} / \mathrm{a}$ & $\mathrm{n} / \mathrm{a}$ \\
\hline II $^{10}$ & 5.1 a 0.7 & $\mathrm{n} / \mathrm{a}$ & $\mathrm{n} / \mathrm{a}$ & $\mathrm{n} / \mathrm{a}$ \\
\hline III & 6.2 a 5.5 & 3.5 a 1.9 & 0.9 a 2.1 & $\mathrm{n} / \mathrm{a}$ \\
\hline IV & 8.1 a 7.4 & 6.4 a 5.7 & 0.9 a 0.3 & $\mathrm{n} / \mathrm{a}$ \\
\hline V & 8.1 a 7.4 & 6.4 a 5.7 & 3.7 a 3.0 & 2.0 a 1.3 \\
\hline VI & 7.9 a 7.2 & 6.2 a 5.5 & 3.5 a 2.8 & 1.8 a 1.1 \\
\hline
\end{tabular}

\subsubsection{Detector de Silicio del Blanco}

El detector se compone de cuatros planos divididos en dos grupo de dos planos cada uno [31]. Cada uno de los planos se compone de una placa de silicio con 2048 canales, con 300 micrones de ancho y un área activa de 5 centímetros cuadrados, el ancho de los canales es de 25 micrones. Los planos fueron construidos por la compañía MICRON SEMICONDUCTOR Limited de Inglaterra.

Los pares de planos están inclinados uno a +45 grados y el otro a -45 grados con respecto a la

\footnotetext{
${ }^{10}$ Los cambios realizados entre el periodo 1 y 2 fueron en la configuración de los detectores no en el area del blanco.
} 
horizontal. Cada plano tiene coordenadas desde el mismo punto de vista que el de los SSD. Los planos fueron colocados de una forma que al desmontar uno no afectara la posición de su pareja. Esta decisión de diseño fue por razones de mantenimiento, además se podía desconectar la electrónica conectada al plano sin afectar su posición. Se poseían cinco planos, cuatro fueron utilizados y el quinto fue el repuesto en caso que algunos de los otros fallaran. La disposición de los planos producía muy buena resolución en la posición de los planos X-Y.

El funcionamiento de un detector de estado sólido es el siguiente. El mismo consiste de un diodo colocado en "reverse bias" y cuando una partícula cargada pasa por el material semiconductor se produce un movimiento de los electrones, produciendo de esta forma una corriente eléctrica que luego es traducida por la electrónica adicional en una señal. Gran parte de la electrónica asociada a los planos son amplificadores ya que la señal es algo débil cuando es producida. En los demás aspectos la recontracción de trayectorias se hace utilizando las posiciones de los "hits" o impactos en los planos para de esta forma reconstruir una trayectoria. A continuación presentamos un diagrama en el cual esta representado uno de los planos del TSD.

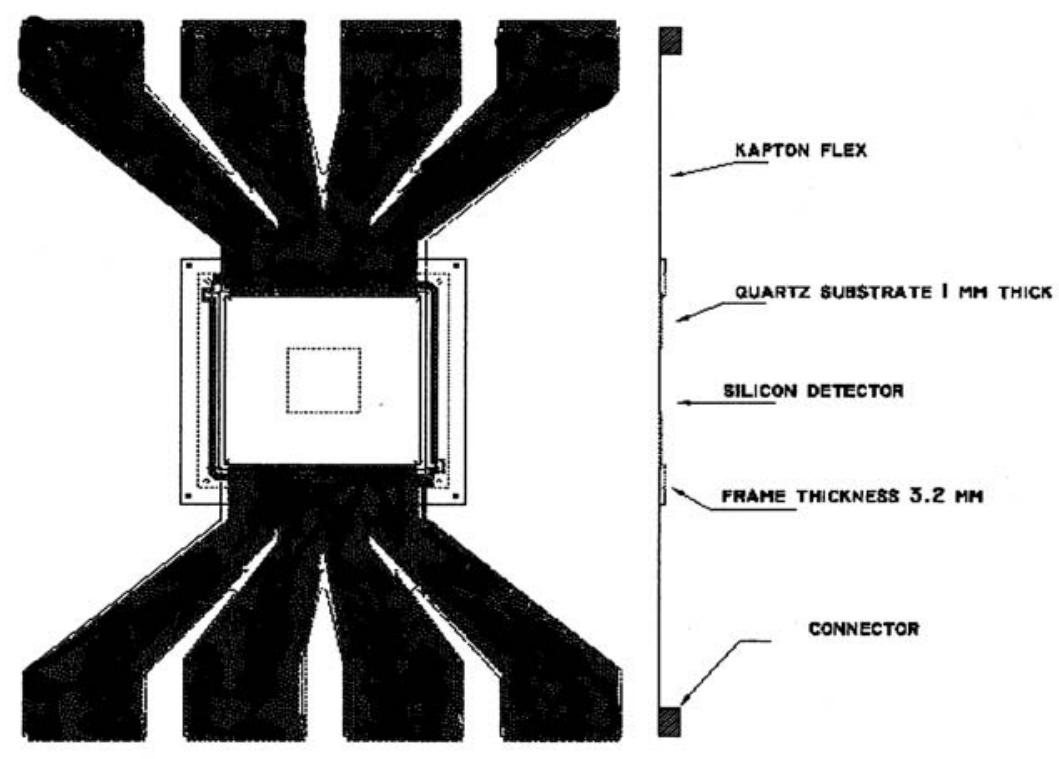

Figura 2.11

Un plano del TSD 


\subsection{Estudio del canal de decaimiento}

\subsubsection{Introducción}

El principal fenómeno estudiado en la física de partículas son los procesos de decaimiento. Un decaimiento es el proceso mediante el cual una partícula pase por un proceso de desintegración que esta regido por una fuerza fundamental se transforma en otras partículas. Una partícula puede decaer dando en el estado final diversas combinaciones de partículas hijas. La probabilidad es lo que rige por cuál canal es que va a decaer. Las probabilidades para las diferentes combinaciones de partículas hijas en el estado final están dadas por la matriz $\mathrm{CKM}^{11}$.

Los decaimientos pueden dividirse por el tipo de partículas hijas que están presentes en el estado final. Tenemos tres categorías, las cuales son hadrónicos, semileptónicos y leptónicos. En la primera categoría tenemos solo hadrónes en el estado final, pueden ser mesones o bariones. En la segunda categoría tenemos hadrónes y leptones presentes. En la categoría final están los leptónicos en estos solo leptones están presentes. En el caso de nuestro análisis los decaimientos que vamos a estudiar son todos hadrónicos.

\subsubsection{Reconstrucción de un decaimiento en FOCUS}

Los datos de FOCUS fueron guardados en cintas magnéticas para su posterior uso. La estructura de los datos es en bloques. En un comienzo los bloques, que eran llamados "Raw blocks" correspondían a un detector en particular y solo poseían la señal en la forma más elemental que se podía guardar. Prácticamente correspondía a la información de las incidencias o "hits" de las partículas en los detectores

Existen dos rutinas principales para la reconstrucción de los vértices primarios, estas son Dfree y Dvnucl. Ambas realizan el mismo trabajo pero utilizando diferentes algoritmos. Cada uno de los algoritmos de reconstrucción se utiliza para los diferentes tipos de decaimientos posibles de una partícula que contenga charm. En el caso de un decaimiento hadrónico se utiliza Dvnucl para realizar la reconstrucción. En este caso se posee toda la información de las partículas hijas. El algoritmo de la rutina es crea una trayectoria virtual, para la construcción de ésta lo que se utiliza es el momentum de la partículas hijas. Luego proyectando la trayectoria virtual se busca que esta se intercepte con lo que llamamos trayectorias nucleadas ${ }^{12}$ y entonces formar un vértice

$11 \mathrm{CKM}$ es el ácronimo para Cabibbo-Kobayashi-Maskawa.

12 Se conoce como trayectoria nucleada a aqúellas que no provienen de un vértice de decaimiento o que son de una 
primario. En este proceso se puede encontrar varios vértices primarios, y se escoge el que tenga el nivel de confianza mayor y la mayor multiplicidad.

En el caso de Dvfree los que se hace es que se posee una lista de posibles vértices primarios entonces el usuario decide si va a utilizar el vértice de mayor multiplicidad o el vértice que esta más alejado del vértice secundario. Entonces el en caso de Dvncul su algoritmo de reconstrucción lo que hace es proyectar la suma de los vectores de dirección de las partículas hijas y luego lo proyecta. Se toma el vértice de la lista el cual tenga el menor error en la posición.

El siguiente caso es cuando tenemos un decaimiento semileptónico. En éstos conocemos parcialmente las trayectorias de las partículas hijas ya que una de ellas es imposible reconstruirla con nuestros detectores, esta es la correspondiente a la del neutrino

En el caso de un decaimiento es leptónico solo podemos observar la trayectoria del leptón con carga, porque la otra correspondiente al neutrino no se puede. Para estos casos se utiliza la rutina DNUGG la cual es parecida a DVNUCL.

Para poder eliminar los eventos reconstruidos los cuales provienen de eventos que no contienen charm o reflexiones ${ }^{13}$. Se utiliza dos rutinas que se llaman ISO1 e ISO2. La idea de ellas es aislar el vértice primario.

\subsection{Procesos de selección de eventos}

Como habíamos dicho en las secciones pasadas la cantidad de datos del experimento era algo prohibitiva para analizar todas las cintas cada vez que queramos hacer un análisis. Por esta razón se estableció un sistema por el cual reducíamos la cantidad de cintas magnéticas a analizar. Este proceso lo conocimos como proceso de selección de eventos. En FOCUS este proceso se realizó en varias etapas. La primera se llamó PASS 1. Las posteriores se llamaron SKIM 1 y SKIM $2^{14}$. Todos los procesos tomaron aproximadamente unos dos años en realizarse.

\subsubsection{Pass One}

PASS ONE se llevó a cabo en las facilidades de FERMILAB. Para su realización se utilizaron una seria de "Clusters" o redes de computadoras. La utilización de este tipo de equipo es necesaria por la cantidad de datos que iban a ser analizados y se requería que fueran analizados de la forma más rápida posible. Esto solo se puede lograr si el trabajo en dividido entre el mayor

partícula que contiene charm. Estas son de partículas que se forman cuando el fotón interactúa con algunos de los nucleones del blanco de oxido de berilio.

13 Para saber más sobre las diversas fuentes de señal de fondo lea la seccion 4.2.5 de esta tesis.

14 En ingles Skim quiere decir el proceso por el cual se obtiene la nata de la leche, en otras palabras se saca lo mejor de la leche. 
número de máquinas posible. Una red o "Clusters" cumple con este propósito.

\subsubsection{Skim One}

La siguiente etapa en el proceso de selección de eventos fue llamada Skim One. En esta ocasión los datos se separaron en seis grupos diferentes para que de esta forma diferentes instituciones de la Colaboración realizaran el trabajo. Este proceso se llevó a cabo en dos diferentes instituciones, la Universidad Vanderbilt y la de Universidad de Colorado. Al finalizar este proceso los datos quedaron divididos en seis subgrupos. Cada uno de ellos poseía los datos correspondientes a todos los decaimientos de un solo tipo. Luego estos subgrupos fueron enviados a otras instituciones para una subdivisión más. En la siguiente tabla se presenta los diferentes subgrupos divididos por el tipo de datos que poseían y a las instituciones que le tocó analizarlos. Estos subgrupos fueron llamados superstream.

Tabla 2.12

Descripción de los diferentes "superstream"

\begin{tabular}{|c|c|c|}
\hline Superstream & Tipos de datos & $\begin{array}{c}\text { Institución que realizo } \\
\text { el análisis de datos }\end{array}$ \\
\hline 1 & $\begin{array}{c}\text { Semi-leptónicos } \\
\text { Topología de los vértices } \\
\text { y } \mathrm{K}_{\mathrm{s}}\end{array}$ & Illinois \\
\hline 2 & $\begin{array}{c}\text { Decaimiento raros y } \\
\text { calibración }\end{array}$ & CBPF, Brasil \\
\hline 3 & Bariones & Fermilab \\
\hline 4 & Estados con quarks \\
\hline 5 & livianos & UC Davis \\
\hline 6 & Decaimientos & UC Davis \\
\hline
\end{tabular}

En nuestro trabajo tomamos los datos del SS2 por la razón de que poseían los datos de topología que necesitábamos y el bloque RAWSTG. Esto nos llevó a que tuviéramos que hacer un proceso diferente de selección de eventos, que fue llevado acabo en nuestras facilidades. En el capítulo 4 explicamos como fue llevado a cabo. 


\section{Capítulo 3 Objetivos}

$$
\begin{array}{r}
\text { "La tarea principal del fisico es hallar por el método } \\
\text { Deductivo, leyes sencillas, lo más generales posibles y } \\
\text { A partir de ellas explicar la configuración de la imagen del mundo." } \\
\text { Albert Eistein } \\
\text { Físico alemán }
\end{array}
$$

Nuestros objetivos son:

1. Determinar el porcentaje de partículas $\mathrm{D}^{+}$cuyas trayectorias se puedan reconstruir sus trayectorias utilizando la información del detector de silicio del blanco.

2. Desarrollar algoritmos que incluyan la información del detector de silicio del blanco para la reconstrucción de las trayectorias del $\mathrm{D}^{+}$.

3. Comparar la eficiencia y la resolución de los diversos métodos para determinar la posición de los vértices de decaimiento de las partículas $\mathrm{D}^{+}$. Se comparan los métodos que usan y los métodos que no usan la información del TSD. 


\title{
Capítulo 4 Procedimiento
}

\author{
"Un proceso no puede entenderse deteniéndolo. \\ El entendimiento debe moverse con el fluir del proceso, \\ Debe unirse a él y fluir con él." \\ Frank Herbet \\ Dune(1965)
}

\subsection{Introducción}

En esta sección de nuestro trabajo explicaremos la metodología que utilizamos para poder alcanzar nuestros objetivos. Para estructurar el procedimiento se tomo como referencia los trabajos hechos por las colaboraciones de los experimentos E791 y E657 [34]. Para lograr que el lector entienda se dividirá la descripción del trabajo en tres fases, donde cada una de ella es dependiente de las demás. La primera de las fases fue la de utilización de la simulación de Monte Carlo. Dicha simulación es la herramienta primordial para cualquier trabajo en el área de la física de partículas. Utilizando la simulación pudimos desarrollar los programas de selección de eventos, los programas de análisis y determinar nuestras eficiencias. Un aspecto que debemos resaltar es la estructura de programación en FOCUS. Ésta permite al usuario tanto utilizar el programa de análisis con datos generados por la simulación de Monte Carlo, como para analizar los datos experimentales. De esta forma se evitan los errores que se puede producir al utilizar diferentes programas los diferentes análisis.

La segunda fase fue la selección de eventos, en ésta se redujo la cantidad de eventos a analizar. Realizamos dos procesos de selección de eventos, en el primero redujimos de 110 cintas magnéticas (unos $528 \mathrm{~Gb}$ de información) a solo 36 cintas. Esta cantidad de datos representaba una quita parte de los datos de FOCUS. En el segundo proceso se redujo el número de cintas a solo unas ocho. En los datos finales poseíamos un conjunto que tenía información del decaimiento $D^{+} \rightarrow K^{-} \pi^{+} \pi^{+}$. Este decaimiento fue escogido porque es uno bien estudiado y de gran estadística. En la siguiente figura esta el diagrama de Feymann para este decaimiento. Un diagrama de Feymann de este tipo presenta los procesos que se ven envueltos en las transformaciones de los quarks. 


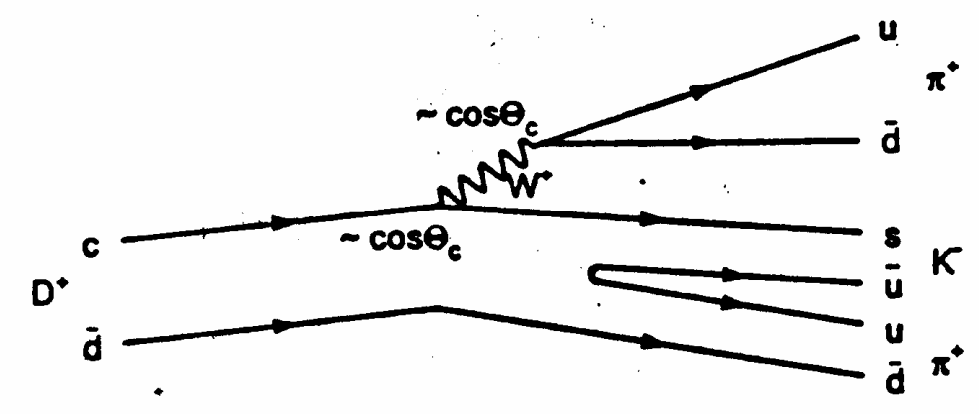

Figura 4.1

Diagrama de Feymann para el decaimiento $D^{+} \rightarrow K^{-} \pi^{+} \pi^{+}$

La tercera fue el análisis de los datos experimentales. El análisis comprendió estudiar relaciones de señal-ruido, análisis de error, entre otros.

A continuación se hace el siguiente desglose del trabajo:

1- Se diseño los programas de selección de datos y reconstrucción de vértices (estos son los llamados programas de análisis), tomando en consideración el decaimiento que se deseaban estudiar. En nuestro caso era un canal dorado, en inglés conocido como un "Golden Mode". El propósito fue ver como nuestras técnicas funcionaban en el proceso de reconstrucción de vértice.

2- Utilizando la simulación de Monte Carlo pudimos estudiar las siguientes cosas:

a- Se calculo las eficiencias. Para los diferentes conjuntos cortes.

b- Se determino nuestras incertidumbres para los diferentes métodos de reconstrucción.

c- Se obtuvo la optimización de los cortes de selección de eventos.

d- Los utilizamos para probar nuestros programas de selección. Esta parte era muy importante porque nuestro trabajo con los datos experimentales dependía mucho de cuan buena era la selección de los eventos.

3- Utilizando los datos experimentales se realizaron los siguientes análisis:

a- La relación señal-ruido para diferentes cortes

b- La relación del "yield" versus cortes.

c- La relación "signal to noise" versus "yield".

d- La distribución de los errores en las posiciones de los vértices primarios y secundarios y en la 
distancia entre ambos.

e- La distribución de los niveles de confianza de los vértices primario y secundario.

f- La correlación entre las diferentes variables, para determinar dependencia o independencia entre las mismas.

\subsection{Simulación de Monte Carlo}

\subsubsection{Procedimientos previos}

El primer paso que realizamos fue determinar qué decaimiento se iba a estudiar para determinar si el añadir la información del TSD mejoraba la reconstrucción de eventos. El decaimiento tenía que tener las siguientes características para que pudiera ser considerado.

a- La partícula madre tenía que durar lo suficiente para que pudiera dejar señal en alguno de los planos de TSD.

b- Al reconstruir las trayectorias de las partículas hijas teníamos

que obtener la mayor información posible.

c- Por ultimo la partícula madre tenía que tener carga eléctrica por que solo éstas eran las partículas que podía detectar el TSD.

d- El decaimiento escogido tenía que tener la mayor estadística posible, es decir la razón de canal de decaimiento tenía que ser alta.

Para cumplir con lo primero utilizamos el paso promedio libre como método de estimación. Si la partícula madre no dura lo suficiente para que pase por alguno de los planos del TSD no se podrá hacer el análisis que queremos. El PPL (paso promedio libre) de una partícula se obtiene de la multiplicación de la velocidad de la partícula por la vida media de la partícula en marco de referencia del laboratorio, $\tau \gamma$, donde $\gamma=E / m$, donde $E$ y $m$ son la energía y masa de la partícula respectivamente. La ecuación que resulta de la multiplicación es la siguiente:

$$
\Delta Z=c \tau \gamma
$$

Donde tenemos que recordar que en nuestra notación $c$ es la velocidad de la luz en el vacío. Esta ecuación nos da una buena aproximación para nuestros propósitos. En la siguiente tabla presentamos para los mesones con charm cuáles son sus PSS a una energía de $180 \mathrm{GeV}$ para el 
rayo de fotones. Para nuestros cálculos estimamos la energía de las partículas con charm en unos $90 \mathrm{GeV}$.

Tabla 4.1

Información sobre las partículas candidatas para estudio que contienen charm

\begin{tabular}{|c|c|c|c|}
\hline Partícula & $\tau(s)$ & Masa $(\mathrm{GeV})$ & $\Delta Z$ \\
\hline$D^{+}$ & $1.00 \times 10^{-12}$ & 1.869 & $1.5 \mathrm{~cm}$. \\
\hline$D^{0}$ & $4.2 \times 10^{-13}$ & 1.865 & $0.608 \mathrm{~cm}$. \\
\hline$D_{s}^{0}$ & $4.5 \times 10^{-13}$ & 1.970 & $0.617 \mathrm{~cm}$. \\
\hline$\Lambda_{c}^{+}$ & $2.0 \times 10^{-13}$ & 2.285 & $0.236 \mathrm{~cm}$. \\
\hline
\end{tabular}

Utilizando los criterios que establecimos al principio de esta sección la reconstrucción de las partículas hijas tenía que ser lo más precisa posible. Esto nos lleva a que el decaimiento tiene que ser hadrónico. Las partículas de más fácil reconstrucción con los detectores de nuestro espectrómetro fueron los hadrónes y los leptones con carga, en especial los muones; ya que se poseía un detector especialmente construido para su detección. Esto reduce nuestro grupo de posibles canales de decaimiento a solo el grupo de los hadrónicos. Los decaimientos pueden dividirse de acuerdo al tipo de partículas en el estado final. Existen tres posibilidades, un decaimiento hadrónico. En éste todas las partículas hijas son mesones, bariones o combinación de ambas. En un decaimiento semileptónico tenemos un hadrón más uno o más leptones. Por último tenemos un decaimiento leptónico, en éste solo hay leptones presentes. Este último de decaimiento estadísticamente es bien baja su incidencia. El problema con los decaimientos semileptónico y leptónicos es que en ambos casos está presente un neutrino ${ }^{15}$. Los neutrinos son las partículas más difíciles de detectar, el hecho de que su masa es casi cero y no poseen carga eléctrica hace que casi no tengan interacciones con otras partículas. Por consiguiente un decaimiento que contenga un neutrino en el estado final no nos es útil, porque queremos tener la información de la trayectoria de todas las partículas hijas. Un factor también importante es que la vida media de las partículas hijas tiene que ser lo suficientemente larga para que se pudiera hacer una buena reconstrucción de sus trayectorias sin la necesidad de hacer vértices adicionales para lograrlo. Esto no reduce a que los decaimientos tenían que tener preferiblemente kaones y piones

15 Exciten decaimientos leptónicos en los cuales tenemos $X \rightarrow l^{+} l^{-}$donde son un par conjugado, pero la posibilidad de este tipo de decaimiento es bien baja casi cero y por esta razón no los consideramos en nuestro análisis. 
en el estado final. Si uno hace un pequeño análisis como el anterior tenemos que los decaimientos con piones y kaones en el estado final eran los mejores. Esto nos lleva a tener dos partículas madre candidatas que eran el $D^{+}$y el $D_{s}{ }^{+}$. Si entonces analizamos la geometría del área del blanco encontramos que necesitamos una partícula que viaje al menos unos 1.2 centímetros aproximadamente, por consiguiente descartamos el $\mathrm{D}_{s}{ }^{+}$como partícula madre para nuestro análisis. Esto nos reduce al siguiente grupo de canales de decaimiento como candidatos, $D^{+} \rightarrow K^{-} \pi^{+} \pi^{+}, D^{+} \rightarrow K^{-} K^{+} \pi^{+}$y $D^{+} \rightarrow \pi^{-} \pi^{+} \pi^{+}$. De estos el más abundante es el $D^{+} \rightarrow K^{-} \pi^{+} \pi^{+}$que es un canal dorado. Es también es un decaimiento bien estudiado y casi siempre es utilizado para analizar las razones de canal de decaimiento de otros decaimientos. En la siguiente tabla están las razones de canal de decaimiento de varios canales.

Tabla 4.2

Razones de canal de decaimiento para los diferentes canales de $D^{+}$

\begin{tabular}{|c|c|}
\hline Canal de decaimiento & Razón de canal de decaimiento \\
\hline & $(9.0 \pm 0.60)$ \\
$D^{+} \rightarrow K^{-} \pi^{+} \pi^{+} \mathrm{CF}$ & $(8.5 \pm 0.8)$ no resonante \\
\hline$D^{+} \rightarrow K^{-} K^{+} \pi^{+}$ & $(8.7 \pm 0.7) \times 10^{-3}$ \\
\hline & $(3.6 \pm 0.4) \times 10^{-3}$ \\
$D^{+} \rightarrow \pi^{-} \pi^{+} \pi^{+}$ & $(2.2 \pm 0.4) \times 10^{-3}$ no resonante \\
\hline
\end{tabular}

\subsubsection{Simulación del canal de decaimiento}

El MCFOCUS es la simulación total del experimento. La simulación de un evento se completa en cuatro etapas. La primera de ella es la generación, en ella se simula el proceso de fotón-gluón y los diferentes canales de decaimiento con sus masas, vida media y momentum. Esta generación simulada incluye todas las partículas que se generan en el blanco experimental y en los radiadores. La segunda parte simula la interacción de las partículas generadas con los detectores. La idea principal es crear los datos que corresponde a los "raw data" en los datos reales. La tercera y última etapa corresponde a la reconstrucción de los eventos. Ésta es la sección que el usuario utiliza su programa de análisis. Este es el mismo que va a utilizar para analizar los eventos experimentales o reales, de esta forma se evita añadir errores por utilizar un programa 
diferente en los procesos de reconstrucción.

La generación de un evento puede ser resumido en los siguientes pasos. El algoritmo utiliza Pythia para producir una lista de productos de la interacción del vértice primario. En esta producción está incluida la información del cuatri-momentum de la interacción y luego LUND devuelve las listas de las identidades de las partículas producidas.

El programa funcionaba de la siguiente forma. Primero se buscaba en el evento todas las trayectorias candidatas a ser un kaón y luego se buscaban los dos piones. Se examinaba si las tres trayectorias escogidas eran candidatas a ser un kaón y dos piones tenían un vértice en común. Este vértice tenía que tener un nivel de confianza mayor del 1\%. Las cargas de las partículas tenían que tener las siguientes combinaciones. Si era el canal dorado, la carga del kaón era diferente a la de los piones y las de los piones la misma. Por otro lado en el caso del canal doblemente suprimido la carga de los piones es diferente y la del kaón igual a la de uno de ellos. Luego de reconstruir el vértice secundario se determinaba la masa invariante, esta tenía que estar entre $1.75 \mathrm{GeV}$ y $1.96 \mathrm{GeV}$. Tenemos que recordar que la masa de la partícula $\mathrm{D}$ es de $1.86 \mathrm{GeV}$. El rango es para cubrir lo más posible los extremos de la distribución guassiana de la señal de masa.

Para reconstruir el vértice primario se siguió la siguiente metodología. Primero se intentaba la reconstrucción con DVFREE. La rutina de DVFREE funcionaba de la siguiente forma. Luego de ser reconstruido el vértice secundario se procedía a buscar el vértice primario más indicado tomando como criterios dos características; la multiplicidad y cual era el más alejado. Se sabe por resultados estadísticos que los vértices primarios con mayor multiplicidad son los que al ser reconstruidos tiene las mayor probabilidad de ser vértices donde se produjo una partícula que contenga charm. En el caso de los que tenga la mayor distancia entre el vértice primario y secundario también ocurre lo mismo. Tomando estas dos consideraciones es que se toma el vértice primario, de esta forma es que toma el primer vértice primario. Al ya tener este vértice se utiliza en la rutina DVFITSD para poder crear la información necesaria para con la utilizar los datos del TSD poder reconstruir el vértice primario. La rutina DVFITSD integra la información del TSD en la reconstrucción del vértice primario creando con estos la trayectoria de la partícula madre. Tenemos que aclarar algo al lector la rutina no determina un nuevo vértice primario, sino que provee información necesaria para la reconstrucción del vértice. Luego la trayectoria reconstruida es utilizada como un objeto que se provee a la rutina de DVNUCL para que esta proceda a realizar la reconstrucción del vértice primario. En el caso de DVNUCL funciona de una forma algo diferente a DVFREE. Al reconstruir el vértice secundario se puede proyectar un vector de momentum que fue calculado por la suma de los vectores de momentum 
de las partículas hijas. Este es proyectado entonces se busca en la lista de vértice primarios candidatos el vértice que esta más cerca de la trayectoria. Al finalizar este proceso se obtiene el vértice primario reconstruido con la información del TSD, porque se integro la información de la trayectoria de la partícula madre. En esta sección del programa obtenemos dos posiciones de vértice primario, una que se obtuvo con DVFREE y otra que se obtuvo con DVNUCL utilizando la información del TSD.

La segunda sección del programa tenía la siguiente secuencia. Primero se reconstruía el vértice primario utilizando DVNUCL. De tener éxito utilizando DVFITSD para proceder igual que lo hicimos en la parte anterior reconstruyendo la trayectoria de la partícula madre y se reconstruía de nuevo utilizando DVNUCL. La información que se guardaba en cada uno de las reconstrucciones está contenida en la tabla 4.3. Una aclaración importante que debemos hacer es que en la simulación de Monte Carlo no podemos generar bloques de datos del tipo RAW de forma directa. Para poder simular esta información lo que se utilizaba cuatro rutinas. Las dos primeras dos hacia el proceso de añadir la información correspondiente al TSD en un bloque artificial de RAWSTG ${ }^{16}$. Las otras dos rutinas realizaban el proceso de utilizar esta información "artificialmente" creada. En la siguiente tabla presentamos los datos que se guardaba por cada uno de los primarios reconstruidos.

\section{Tabla 4.3}

Variables que se guardan en cada determinación de un vértice primario

\begin{tabular}{|c|}
\hline Variables \\
\hline Posición en cada uno de los ejes \\
\hline Error en la posición de cada uno de los ejes \\
\hline Multiplicidad del vértice primario \\
\hline Nivel de confianza del vértice \\
\hline
\end{tabular}

Cada una de las variables tiene una importancia particular. A continuación vamos a explicar cada una de ellas.

\footnotetext{
${ }^{16}$ El formato de los bloques de datos estaban divididos en dos grupos, los datos originales o RAW y los datos reconstruidos. Un bloque RAW tenía la información directa del detector cuando se corrió el experimento. En el caso de un bloque REC son datos reconstruidos, no necesariamente estos bloque no tenia que corresponder a un detector en particular.
} 
Multiplicidad: esto es referente al número de trayectorias que tiene su origen en el vértice primario.

2. Nivel de confianza del vértice: Cada uno de los vértices esta reconstruido por un algoritmo que tiene asociado un nivel de confianza. Esto hace que no todas las trayectorias se intercepten en el mismo punto en el espacio. Nosotros definimos entonces un volumen alrededor del candidato a vértice. Este volumen tiene forma de elipsoide. La forma se debe a que el error en el eje de $z$ es mayor que en los ejes $x$ y $y$. Los niveles de confianza que pedimos para el vértice mayor de $1 \%$, este valor es el mínimo para eliminar la señal de fondo.

3. Posición del vértice en el eje de $z$ : es la posición donde el vértice primario se formo. Esta posición es a lo largo del espectrómetro.

4. Error en la posición de z: es el error en la determinación de la posición en el eje de $\mathrm{Z}$.

\subsubsection{Utilización de los datos divinos}

En el caso del programa para ser utilizado para el análisis de los datos que provienen de la simulación de Monte Carlo se le añadió una parte para guardar los datos correspondientes al "God's Block". Cuando se genera un evento se crea una serie de datos los cuales se podrían llamar primarios. La simulación toma eso datos y añadiéndoles los ruidos de los detectores y ciertas funciones de incertidumbre se realiza la reconstrucción como si tratara de un dato real.

El método que utilizamos para poder determinar si nuestra reconstrucción era correcta y determinar nuestros errores en la misma, fue simplemente comparar los datos provenientes del "God's Block" con los datos reconstruidos. De ahora en adelante en este trabajo nos referiremos a los datos del "God's Block" como GD y los reconstruidos como los RD. Los datos en que nos concentramos nuestro trabajo fue la posición del vértice primario, en especial la posición de este en el eje de z, la posición de vértice secundario, también primordialmente la posición en el eje de z; la distancia entre ambos ejes y distancia entre el eje direccional de la trayectoria generada versus el vértice primario de los RD.

Nuestra metodología fue la siguiente. Se calculaba la diferencia entre el dato GD y el RD. A esta diferencia expresamos en la siguiente ecuación. Donde las variables son posiciones en el eje de Z para los datos reconstruidos y los datos "God Block".

$$
x_{i}=Z_{G D}-Z_{R D}
$$


La distribución de esta diferencia para cualquiera de las variables debe ser guassiana. Luego determinamos el valor promedio de esta diferencia para un grupo de datos en especial.

$$
\bar{x}_{i}=\frac{\sum\left(Z_{G D}-Z_{R D}\right)_{i}}{n}
$$

El siguiente paso fue calcular la varianza de las muestra, determinada por la siguiente ecuación.

$$
s^{2}=\sqrt{\frac{\sum\left(x_{i}-\bar{x}\right)}{n}}
$$

La varianza de un conjunto de datos es el cuadrado de la desviación típica. Si partimos de que la distribución de los es normal o guassiana tenemos que [36]:

a- $68.27 \%$ de los casos están entre $\bar{x}-\sigma$ y $\bar{x}+\sigma$. b- $95.45 \%$ de los casos están entre $\bar{x}-2 \sigma$ y $\bar{x}+2 \sigma$. c- $99.73 \%$ de los casos están entre $\bar{x}-3 \sigma$ y $\bar{x}+3 \sigma$.

En podemos determinar nuestra incertidumbre por la siguiente ecuación.

$$
\sigma_{m}=\frac{\sigma}{\sqrt{n}}
$$


En nuestro caso podemos decir que $S=\sigma$ asumiendo que la desviación calculada es igual o se aproxima a la desviación de la población total. Entonces si aceptamos el $99.73 \%$ la muestra tenemos que la incertidumbre en el valor medio estaría dado por $3 \sigma_{m}$. Podemos mejorar nuestra estimación de la medida de la incertidumbre si utilizamos el criterio de Chauvenet[34].

Entre los cortes de vértices el más importante era la distancia entre el vértice primario y el vértice secundario dividido por el error en la medida. Esta relación la llamamos $l / \sigma$ y esta dada por la siguiente ecuación:

$$
\frac{l}{\sigma}=\frac{V_{\mathrm{sec}}-V_{p r i}}{\sqrt{\sigma_{p r i}^{2}+\sigma_{\mathrm{sec}}^{2}}}(4.6)
$$

Esta relación es muy útil porque $l$ es dependiente del momentum de la partícula madre. En cambio $l / \sigma$ es independiente del momentum esto lo hace útil para considerarse un corte. Un detalle importante es qué se desea que las variables utilizadas sean los más independientes que se puedan.

Dividimos los cortes de posición en tres grupos. El primer grupo lo llamamos como los cortes fuertes. La posición de vértice z está confinada exactamente a la de los blancos. Los otros dos grupos los llamamos cortes suaves I y suaves II. Éstos eran más anchos en términos del rango de aceptación de la posición del vértice. En el siguiente grupo de tablas presentamos los valores de las variables para los diferentes grupos de cortes.

\section{Tabla 4.4}

Cortes Fuertes para el vértice primario

\begin{tabular}{|c|}
\hline Posición del vértice primario \\
\hline$-7.888 \leq z_{p r i} \leq-7.2130$ \\
\hline$-6.2380 \leq z_{p r i} \leq-5.5630$ \\
\hline$-3.5057 \leq z_{p r i} \leq-2.8307$ \\
\hline$-1.7880 \leq z_{p r i} \leq-1.1130$ \\
\hline
\end{tabular}


Tabla 4.5

Cortes suaves I del vértice primario

\begin{tabular}{|c|}
\hline Posición del vértice primario \\
\hline$-7.8910 \leq z_{p r i} \leq-7.2070$ \\
\hline$-6.2410 \leq z_{p r i} \leq-5.5600$ \\
\hline$-3.5087 \leq z_{p r i} \leq-2.8277$ \\
\hline$-1.7910 \leq z_{p r i} \leq-1.1100$ \\
\hline
\end{tabular}

\section{Tabla 4.6}

Cortes suaves II del vértice primario

\begin{tabular}{|c|}
\hline Posición del vértice primario \\
\hline$-7.888 \leq z_{p r i} \leq-5.5630$ \\
\hline$-3.5057 \leq z_{p r i} \leq-1.1130$ \\
\hline
\end{tabular}

Tabla 4.7

Cortes para el vértice secundario

\begin{tabular}{|c|}
\hline Posición del vértice secundario \\
\hline$-4.0800 \leq z_{s e c} \leq-3.5057$ \\
\hline$-0.0900 \leq z_{p r i}$ \\
\hline
\end{tabular}

Los siguientes cortes fueron establecidos utilizando las características de los Čerenkov.

Tabla 4.8

Limites inferiores de momentum

\begin{tabular}{|c|}
\hline Limites inferiores en el momentum de las partículas hijas \\
\hline$p_{K} \geq 16.2 \mathrm{GeV}$ \\
\hline$p_{\pi} \geq 4.5 \mathrm{GeV}$ \\
\hline
\end{tabular}


Tabla 4.9

Cortes básicos comunes

\begin{tabular}{|c|c|}
\hline Variable & Valor \\
\hline PCL & $>1 \%$ \\
\hline SCL & $>1 \%$ \\
\hline ISO1 & $<1 \%$ \\
\hline ISO2 & $<1 \%$ \\
\hline$P_{D}$ & $>70 \mathrm{GeV} / \mathrm{c}$ \\
\hline
\end{tabular}

En la tabla 4.9 presentamos los principales cortes principales. PCL es el nivel de confianza para el vértice primario, SCL es el nivel de confianza para el vértice secundario. Los cortes ISO1 y ISO2 son correspondientes a el vértice primario. En el caso de ISO1 hace una comparación entre el vértice secundario y el primario con respecto a sus posiciones. Por otro lado el corte ISO2 determina si hay trayectorias del vértice secundario pasando por el vértice primario. El corte $P_{D}$ se determino de forma empírica.

\subsubsection{Optimización de los cortes}

Luego que se definieron los cortes, la idea era saber cual era el mejor conjunto. Para determinar la optimización de los cortes utilizamos la eficiencia de la reconstrucción como criterio. Ésta estaba dada por la siguiente ecuación:

$$
\text { eficiencia }=\frac{N_{\text {obs. }}}{N_{\text {gen. }}}
$$

Donde $N_{o b s}$ fue el número de eventos que nuestro análisis pudo detectar y $N_{\text {gen }}$ fue el número de eventos generados por la simulación. Los criterios para decir que un conjunto era mejor sobre otro conjunto era ver cual tenía la mayor eficiencia, pero esto nos podría haber llevado a un error porque podíamos simplemente aceptar todos los eventos que quisiéramos siendo lo más flexibles posible. Por esta razón necesitábamos otro método. Éste fue simular canales que contribuían a la señal de fondo o ruido y determinar cómo nuestros cortes reducían ésta. 


\subsubsection{Estudio de la señal de fondo}

Entre las posibles fuentes de la señal de fondo podemos distinguir tres principales. La primera son canales similares o reflexiones, éstos son canales de decaimiento que por las incertidumbres de los sistemas de identificación de las partículas podemos asumir que son el canal que deseamos estudiar. Casi siempre son canales que tienen una estadística igual o menor a la del canal de nuestro análisis. Son muy difíciles de remover de la señal de nuestro análisis.

Las otras fuentes de ruido son redispersión y combinatoria. La redispersión ocurre cuando partículas que salieron de la interacción original del fotón con el núcleo interactúan con el material del blanco. En el caso de FOCUS este efecto se redujo al segmentar el blanco. La señal de fondo que se produjo por combinatoria es producto de malas reconstrucciones de los vértices. Ambas señales de fondo son generalmente fáciles de parametrizar, porque se pueden describir por una línea recta o por una función exponencial y están gobernadas por un comportamiento aleatorio. En la siguiente grafica presentamos la estructura general de una señal con ruido.

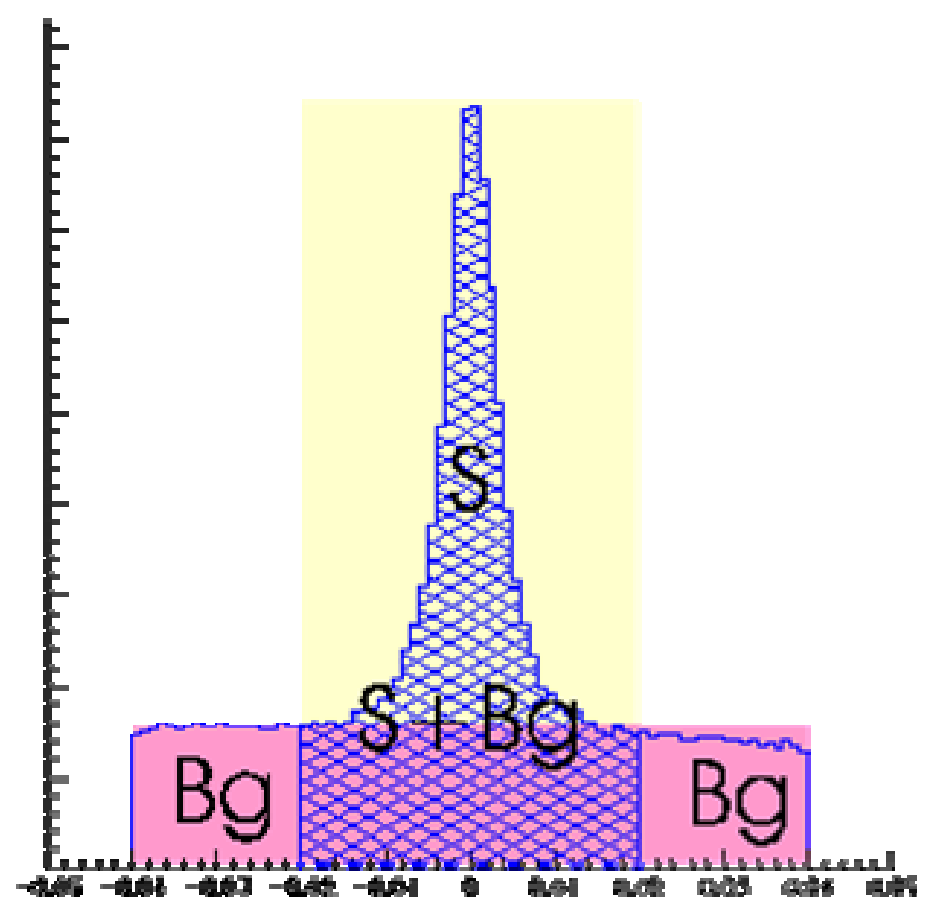

Figura 4.2

Muestra de la estructura de una señal con ruido. 
Nosotros utilizamos la simulación para poder analizar como nuestra metodología eliminaba las señales de fondo y de esta forma poder "saber" como se iba comportar en la señal real. Además nos ayudó a determinar cuales eran los mejores cortes. Para el canal de decaimiento CF el canal de fuente de ruido principal fue el canal $D \rightarrow \pi \pi \pi$. Esto ocurre porque un pión puede ser confundido por un kaón si tiene este suficiente momentum.

Para determinar la eficiencia de nuestros cortes utilizando la señal de fondo utilizamos lo que llamamos RCF (Reduce Channel Factor). Este determinaba el por ciento de eventos que eran eliminados por los cortes. Esto era determinado con las siguientes de ecuaciones.

$$
\begin{gathered}
S=\frac{N_{\text {sobrevient es }}}{N_{\text {original }}}(4.8) \\
R C F=\left(\frac{N_{\text {original }}-N_{\text {sobrevivientes }}}{N_{\text {original }}}\right) \times(\# \text { eventos }){ }^{17}(4.9)
\end{gathered}
$$

\subsection{Proceso de selección de eventos}

En esta sección de nuestro trabajo realizamos nuestra selección de eventos para tener la cantidad exacta de eventos que queríamos analizar, y que cumplieran con nuestros propósitos. Nosotros realizamos dos procesos de selección consecutivos. Como habíamos mencionado en el capítulo 2 los datos de FOCUS primero se dividieron en lo que se llamó "Super stream" y luego se enviaron a diferentes colaboraciones para ser de nuevo subdividido. Por las necesidades particulares de nuestro trabajo tomamos unos de los "Super stream" como punto de partida para nuestra selección, el SS2. Éste contenía el bloque de datos llamado "Global Vertixing" y tenía los datos del TSD en un bloque RAW. Debemos recordar que necesitamos la señal original del TSD para nuestro análisis. Este "Super stream" se componía de una 523 cintas en total. Para

17 Se multiplica por eventos solamente para ser consistente con las unidades. 
propósitos de nuestro trabajo solo utilizamos 110 cintas lo cual representa el $21.0 \%$ de los datos, en otras palabras prácticamente una quinta parte de los datos. Par poder saber si se tomo una muestra representativa del experimento se hizo una gráfica de "RUNNUM" versus número de eventos. La prueba que realizamos fue simplemente que cada período estuviera representado de forma más o menos equitativa. Algo que debemos aclarar que solo los "RUNNUM" mayores de 9725 nos era útiles. Esto se debía a que el TSD estuvo totalmente operacional luego de ese "RUNNUM". A continuación presentamos una serie de gráficas de esta parte de nuestro análisis.

En el primer proceso de selección de eventos se guardaron todos los eventos con tres trayectorias que contenían trayectorias de piones o kaones. (Cualquiera de las combinaciones posibles) Esto se hizo con el propósito de tener un conjunto de datos en el que se pudiera estudiar cualquiera de los decaimientos hadrónicos que contenían kaones y piones. Los cortes utilizados están contenidos en la siguiente tabla.

Tabla 4.10

Cortes de selección utilizados en la primera fase de selección

\begin{tabular}{|c|c|}
\hline Variable & Valores \\
\hline Citadel & $\begin{array}{c}\text { wobs(2)-wobs(3)<0 para el pión } \\
\text { wobs(2)-wobs }(4)>1 \text { y wobs(3)- } \\
\text { wobs }(4)>-2.0 \text { para el kaón }\end{array}$ \\
\hline Istapt & $\begin{array}{c}2 \text { o } 6 \text { para el pión } \\
6 \text { o } 12 \text { para el kaón }\end{array}$ \\
\hline Número de trayectorias & 3 \\
\hline
\end{tabular}

Tabla 4.11

Cortes de selección utilizados en la segunda fase de selección

\begin{tabular}{|c|c|}
\hline Variables & Rango \\
\hline Istatp & 2 ó 6 para el pión \\
& 6 ó 12 para el kaón \\
\hline Número de trayectorias en el evento & $>3$ \\
\hline Nivel de confianza del vértice primario & 0.01 \\
\hline Rango de la masa invariante & $1.78 \mathrm{GeV}$ a $1.95 \mathrm{GeV}$ \\
\hline
\end{tabular}




\subsection{Análisis de los datos experimentales}

\subsubsection{Análisis de los datos del canal dorado}

Para realizar la optimización de los cortes utilizamos lo que se llama la significan cía. Esta nos da una relación entre la señal que deseamos estudiar y la señal de fondo o ruido. Existe también otra relación que es citada en la literatura de habla inglesa como "signal to noise". Mayormente se utiliza para saber cuán buena es una señal. El uso de la significancia es que tiene una relación directa con la distribución de los datos si estos tienen una distribución normal. Ésta está dada por:

$$
\text { significancia }=\frac{S}{\sqrt{S+B}}(4.14)
$$

Para los canales con la relación $B>>S$, la significancia está dada por:

$$
\begin{gathered}
\text { significan cia }=\frac{S}{\sqrt{B}} \\
\text { significancia }_{m c}=\frac{S_{m c}}{\sqrt{S+B}}
\end{gathered}
$$

En nuestro análisis que hicimos incluyó las siguientes determinaciones o procedimientos:

1- La relación señal-ruido para diferentes cortes. Para poder medir el ruido desarrollamos nuestras propias técnicas de estimación del ruido.

2- La relación del "yield" para los diferentes cortes.

3- La relación "signal to noise" versus "yield".

4- La distribución de los errores en las posiciones de los vértices primarios y secundarios y en la distancia entre ambos. 
5- La distribución de los niveles de confianza de los vértices primario y secundario.

6-La correlación entre las diferentes variables, para determinar dependencia o independencia entre las mismas. 


\section{Capítulo 5 Resultados y Conclusiones}

\subsection{Resultados del análisis de la simulación de Monte Carlo}

Al realizar la simulación lo primero que obtuvimos fueron la relación entre la eficiencia y nuestros cortes. El primer conjunto de cortes que probamos fueron los llamados cortes fuertes y posteriormente se fueron probando otros menos rígidos. El medir estar relación nos permitía de una primera instancia hacer una primera determinación de cuales eran los cortes más eficientes para un análisis posterior de los datos experimentales o reales y determinar cual era el mejor método de reconstrucción. En las siguientes graficas se presenta las eficiencias versus $l / \sigma$ para los diferentes grupos de cortes.

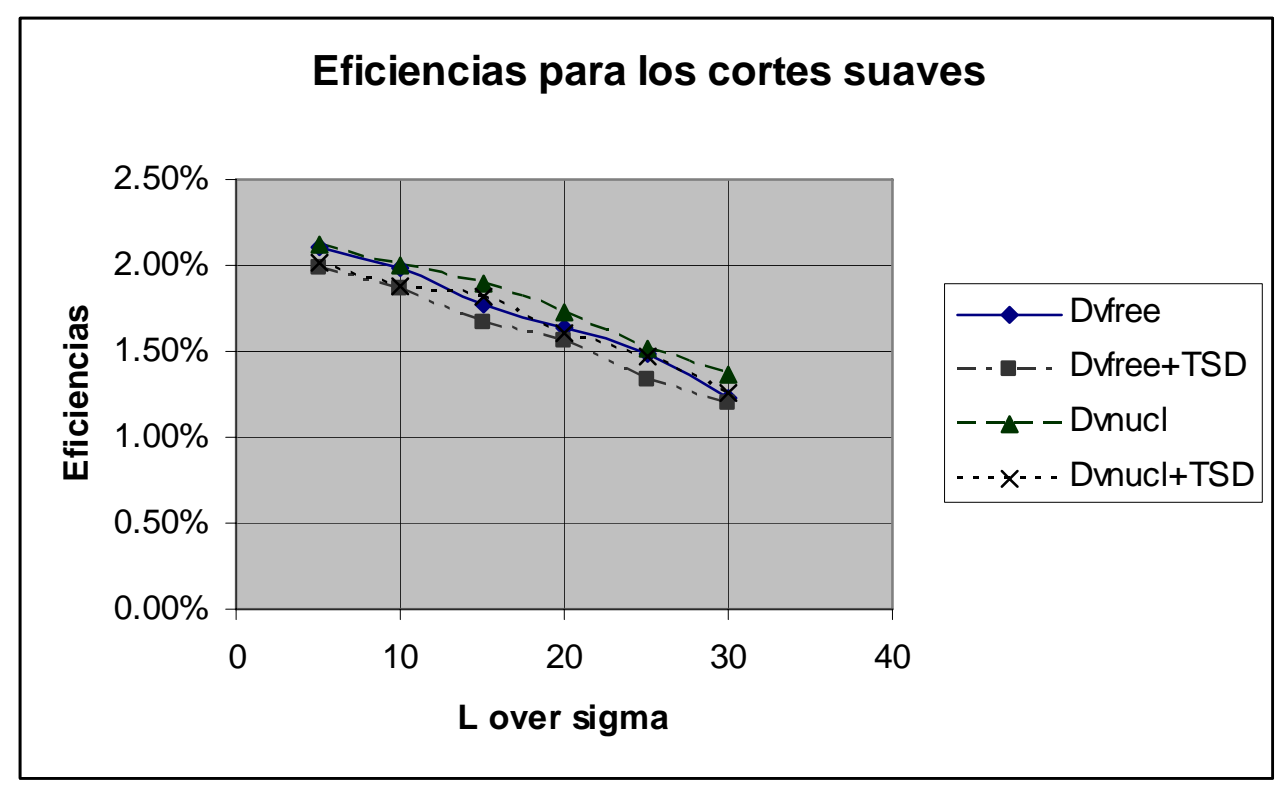

Figura 5.1

Eficiencias para los cortes suaves para los diferentes métodos de reconstrucción

Si se observa con detenimiento se puede observar que el método que utiliza Dvnucl es más eficiente que los demás, inclusive cuando se añade la información del TSD. Para que el lector pueda entender las graficas vamos explicar la leyenda. Los datos que aparecen bajo DVFREE son los correspondientes a los que fueron obtenidos al utilizar dicha rutina. Los datos que aparecen bajo DVNUCL (I) son lo que se utilizó dicha rutina pero utilizando la información del TSD para la reconstrucción del vértice primario. Los datos que aparecen bajo DVNUCL son los que se utilizó esta rutina pero sin utilizar la información del TSD, por último los que aparecen 
bajo DVNUCL (II) son los datos reconstruidos con la información del TSD.

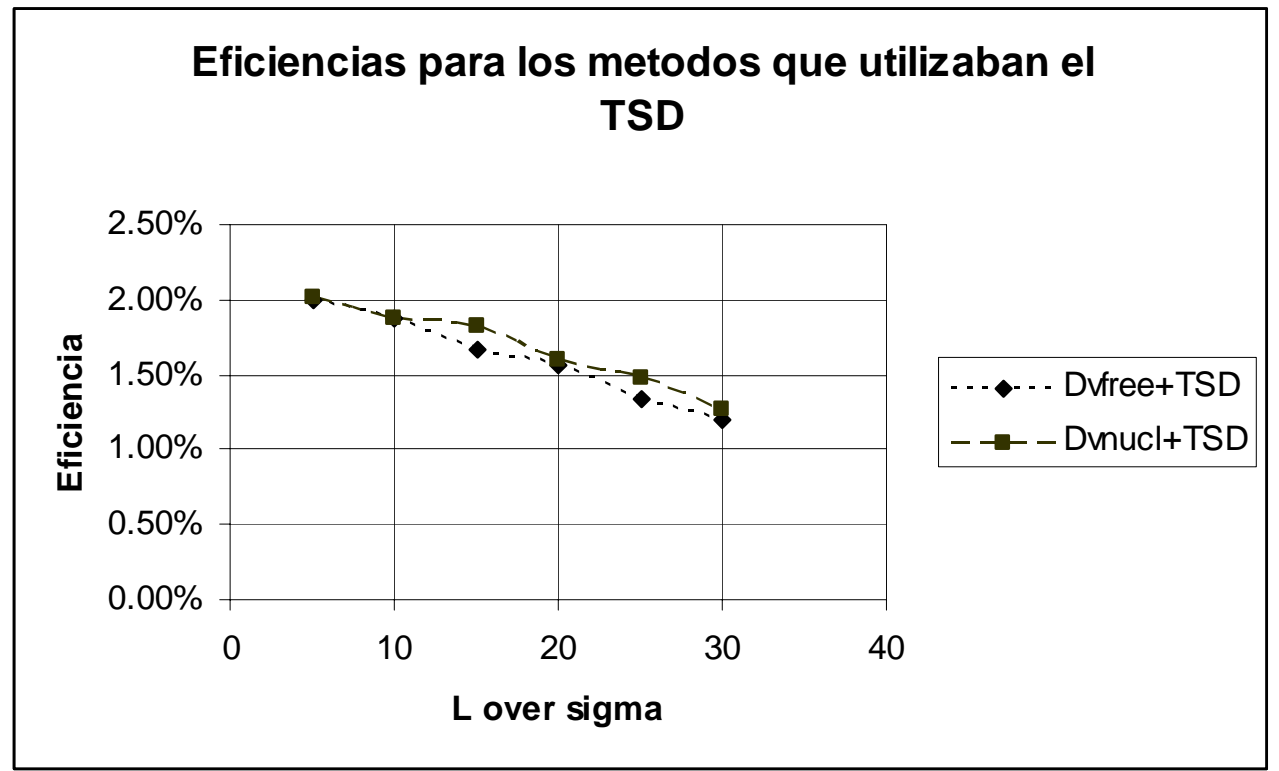

Figura 5.2

Eficiencias para los cortes suaves en los que se utilizo la información del TSD

Si se observa las tendencias se mantuvieron iguales. Tomando esto como un hecho llegamos a la conclusión de que la metodologías de reconstrucción no están afectadas por el tipo de cortes que se utilizó. Entonces la idea es utilizar otros criterios para determinar si la metodología funciona.

Utilizando la gráfica anterior podemos concluir lo siguiente:

1. El método de añadir la información del TSD nos mejoró las eficiencias en un promedio.

2. Para los cortes más fuertes los cambios no fueron tan notables.

3. El utilizar la reconstrucción del Dvfree y Dvfree+TSD combinadas no crearon resultados significativos.

4. El cambio en las eficiencias entre Dvnucl y Dvnucl+TSD fue de un $4 \%$ en promedio. 
A continuación presentamos un grafica de parametrización para la señal de masa reconstruida con Dvfree.

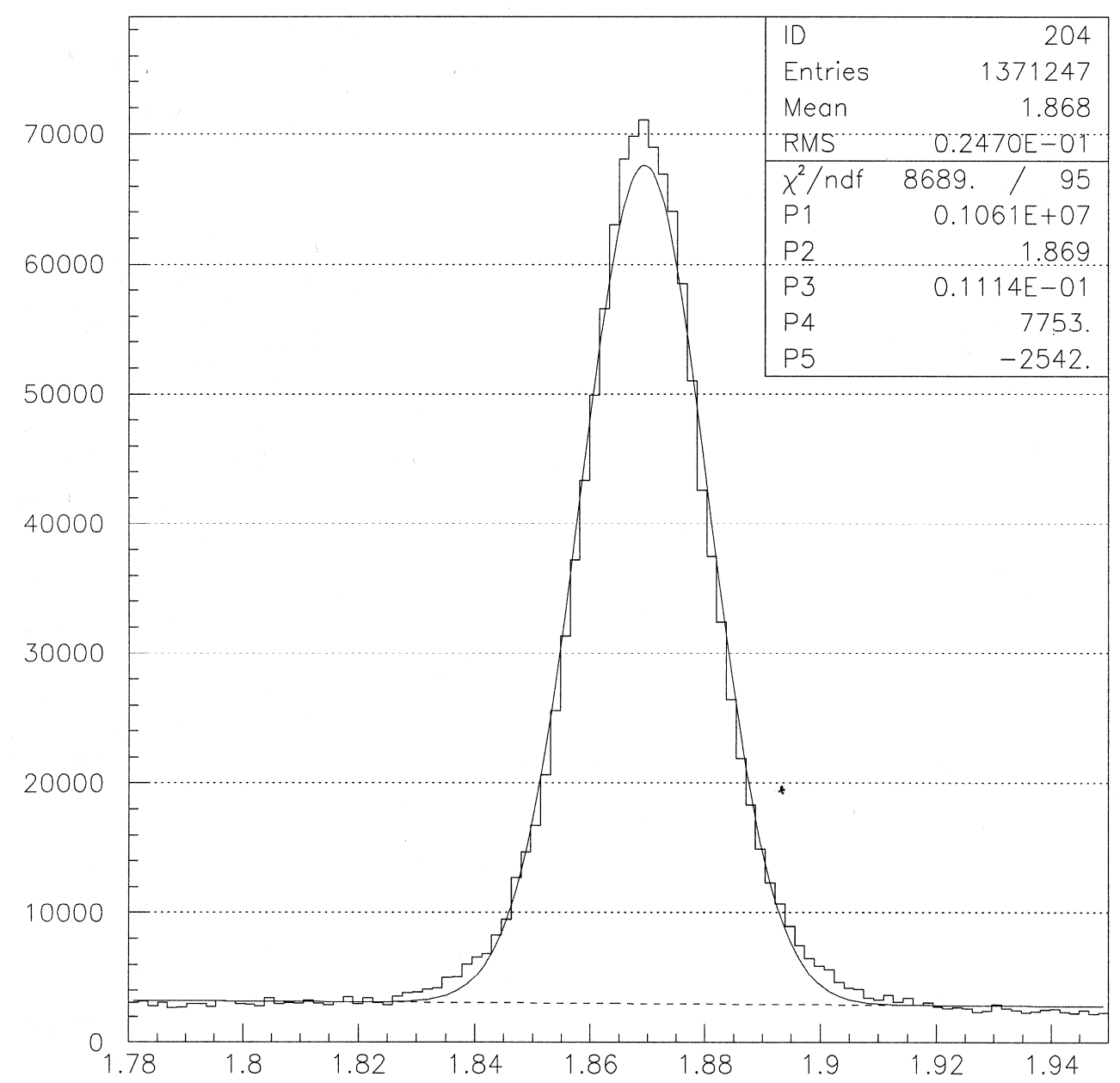

Figura 5.3

Señal de masa reconstruida con Dvfree

Un dato importante era ver la diferencia entre la posición del vértice primario reconstruido con el generado. En la grafica que aparecen en la figura 5.5 vemos la distribución de esta diferencia para los cortes suaves. También se compara la reconstrucción de la señal de masa correspondiente a los datos generados por la simulación y los datos reconstruidos por el programa de análisis (figura 5.6). Estos dos análisis fueron importantes porque ésta era la única forma por la cual se podía saber que la metodología de reconstrucción estaba correctas o en otras palabras, si se estaba mejorando el método de reconstrucción. 


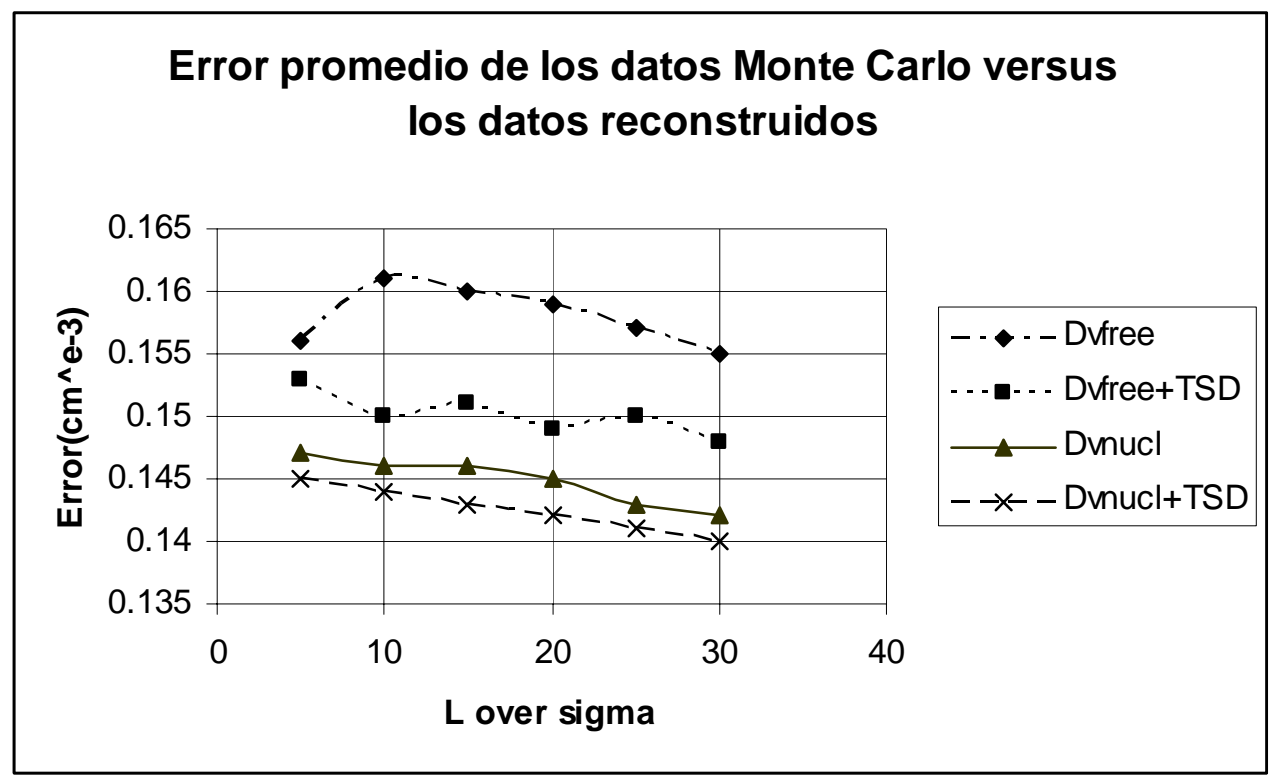

Figura 5.4

Diferencia promedio para la posición del vértice primario de los datos reconstruidos y los generados.

Llegamos a la conclusión de que el mejor método es utilizar Dvnucl y luego añadir la información del TSD. Este da una mejor reconstrucción del vértice primario.

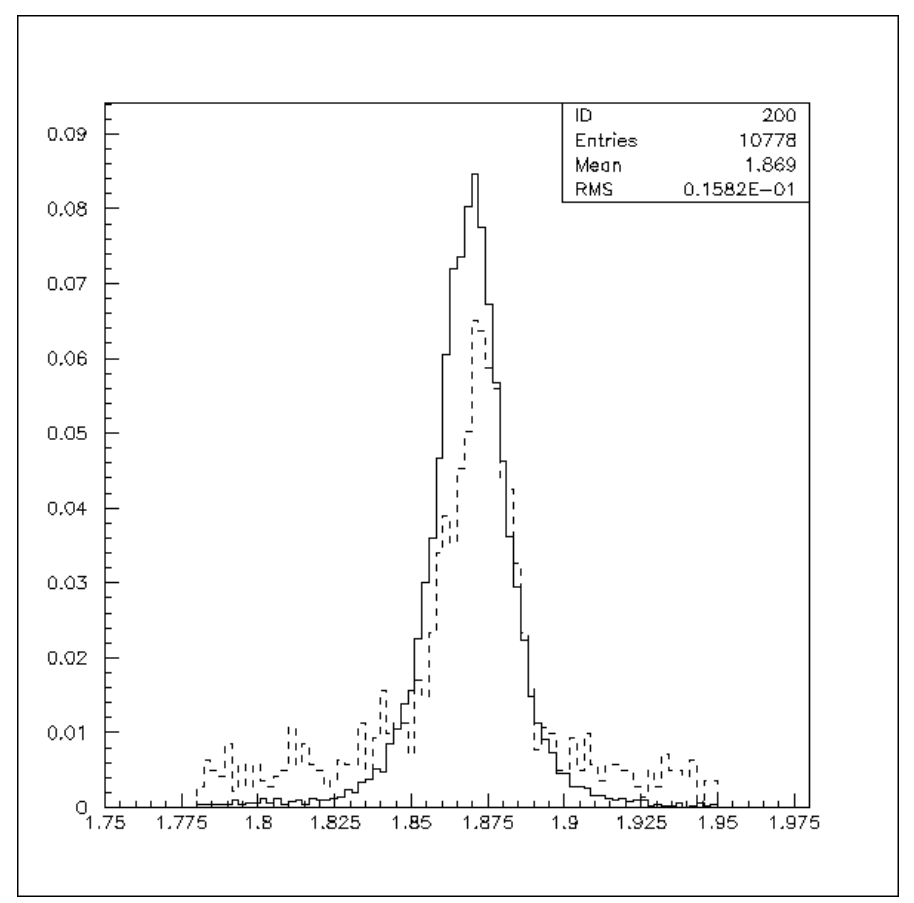

Figura 5.6

Diferencia promedio para la masa

de los datos reconstruidos y los generados utilizando Dvnucl 


\subsection{Resultados del análisis de los datos experimentales}

En el análisis de los datos experimentales nosotros tuvimos dos grupos principales de resultados. El primer grupo fue el estudio de "yield" versus $l / \sigma$ y el siguiente grupo fueron sobre el estudio de $\mathrm{S} / \mathrm{N}$ versus $l / \sigma$. A continuación se presenta un grupo de grafica de rendimiento versus cortes ( figura 5.7).

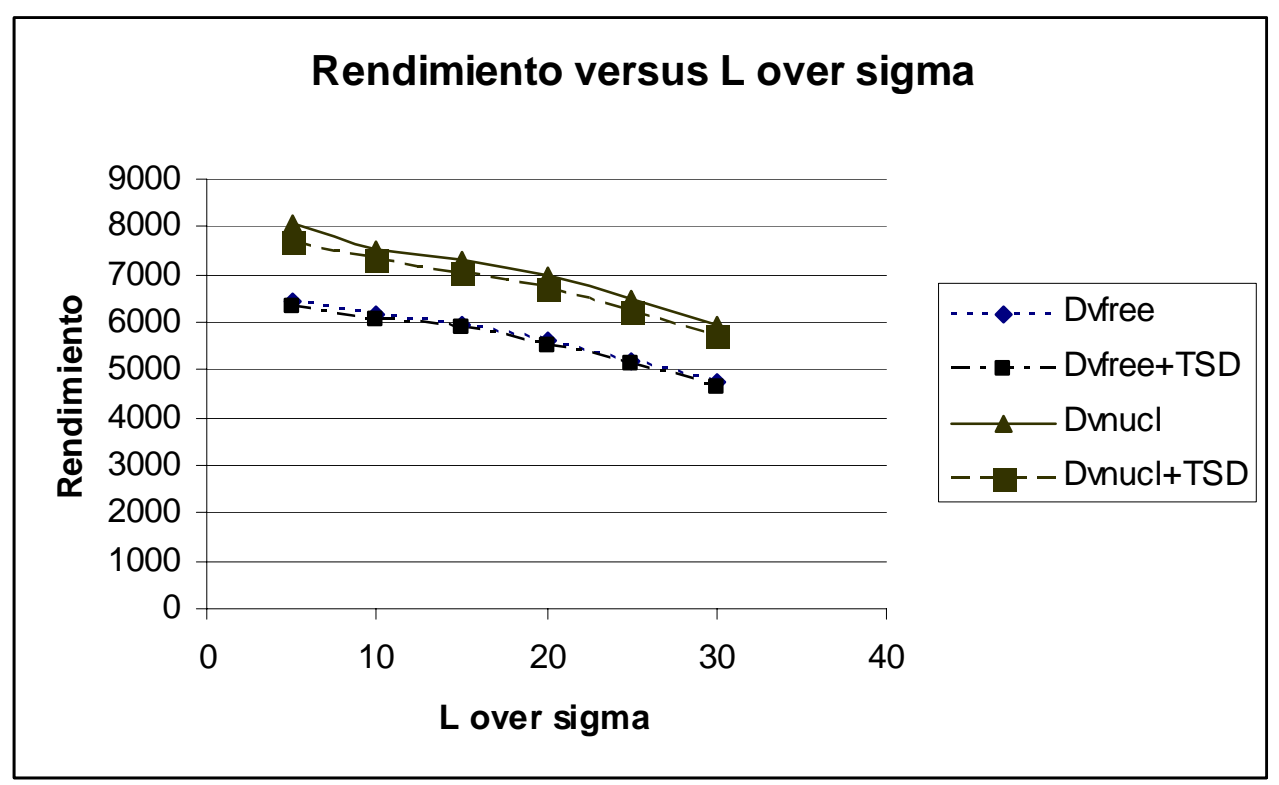

Figura 5.7

Rendimiento versus cortes para los cortes suaves

Si se observa tenemos un resultado consistente con los resultados de la simulación de Monte Carlo. El siguiente análisis fue el comparar cuánto ruido reducíamos utilizando cada una de las metodologías. Cuando se aplica un corte se reduce el ruido, pero también se elimina algo de la parte correspondiente a la señal. Lo que se trata de encontrar es el grupo de cortes en los cuales se reduzca la mayor cantidad posible de ruido pero a la vez perder la menor cantidad de señal posible. En el caso de la simulación de Monte Carlo se pueden producir canales de decaimiento los cuales producen ruido a nuestra señal de análisis. Esto nos es muy útil porque podemos probar si nuestros programas de selección y nuestros cortes reducen este tipo de ruido. En el siguiente grupo de graficas se presentan los diferentes graficas correspondientes a esta parte del análisis. 


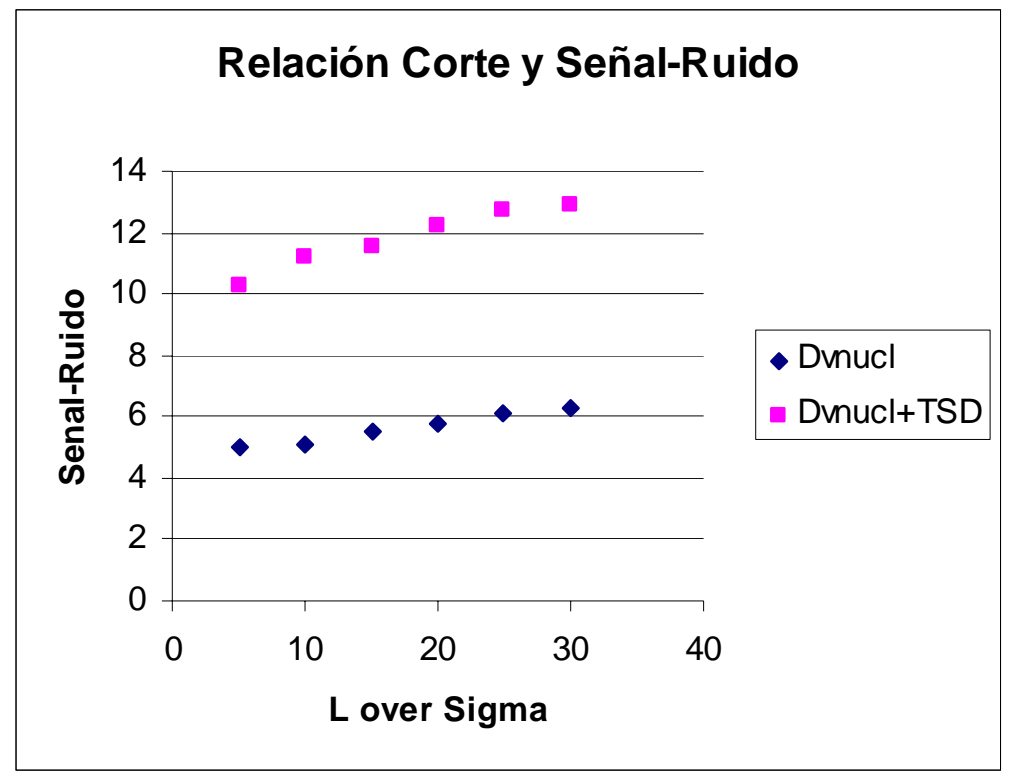

Figura 5.8

Relación de los cortes versus razón señal-ruido

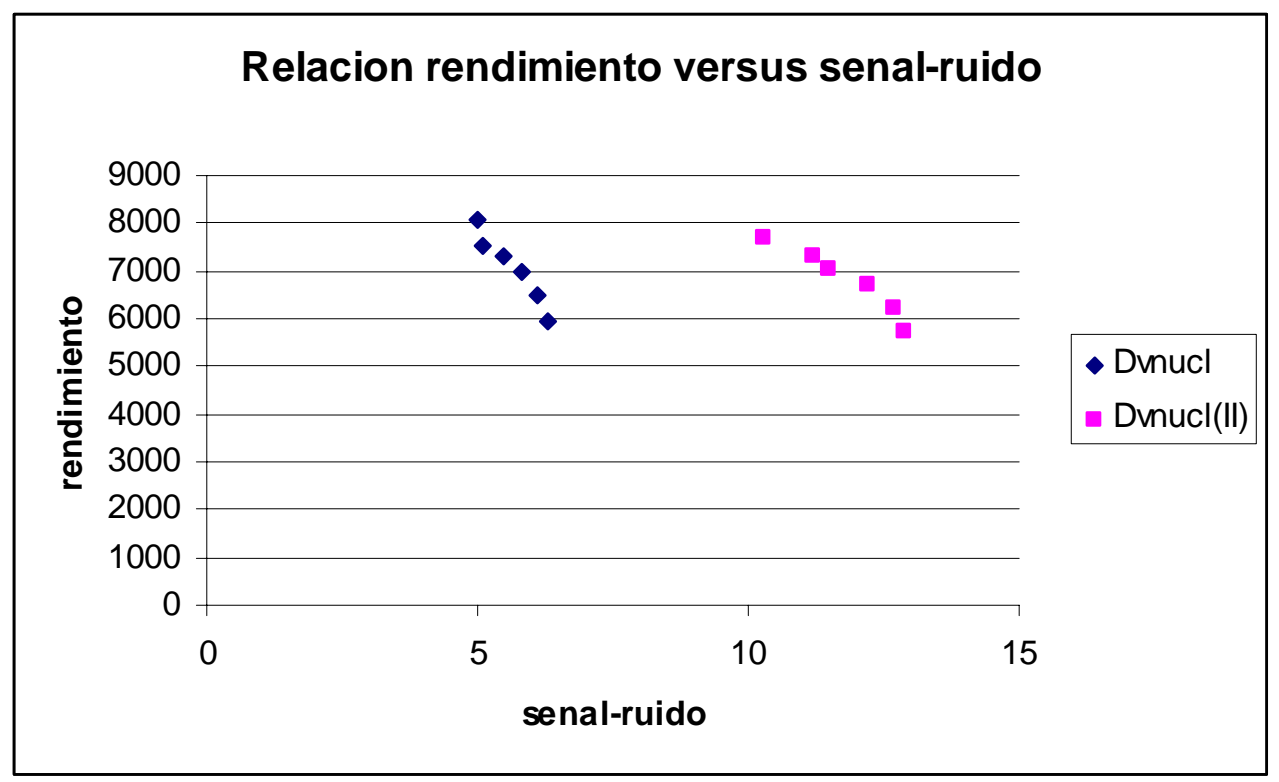

Figura 5.9

Relación rendimiento versus señal-ruido

Si se observa tenemos una mayor señal cuando se utiliza la metodología de Dvnucl con la información de TSD. 


\subsection{Conclusiones}

Tomando en cuenta todos los resultados anteriores presentados podemos llegar a las siguientes interpretaciones:

1.El método de Dvnucl para decaimientos hadronicos es superior que el método de Dvfree y esta diferencia se agudiza cuando se añade la información del TSD.

2. Al utilizar el TSD solo se pierde en promedio el 10\% de la señal.

3. Al utilizar el TSD se reduce en promedio el ruido de fondo en un $60 \%$

4. Al utilizar la metodología de Dvnucl se obtiene en promedio una relación señal-ruido de 5.63 y al utilizar la metodología Dvnucl+TSD se obtiene una relación señal-ruido de 11.8. Esto quiere decir que se duplico la relación señal-ruido. 


\section{Capítulo 6: Sugerencias}

De este trabajo podemos hacer las siguientes sugerencias:

1.Añadir de forma permanente en la simulación de Monte Carlo el detector del TSD.

2. Utilizar esta metodología para otros tipos de análisis como decaimientos semileptónicos y leptónicos.

3. Mejorar la subrutina que añade la información del TSD o incluirla en la metodología de forma directa. 


\section{Bibliografía}

1. Serway, Raymond A., Moses, Claement J. y Moyer, Curt A. 1997. Modern Physics, Saunders College Publishing, Fort Worth, USA 688 pp.

2. Abner, Shimony. 1989. Conceptual foundations of quantum mechanics. En: Paul Davis (ed.) The New Physics, pp. 373-395. Cambridge Universtiy Press Cambridge, Cambridge, United Kingdom.

3. Strange, Paul. 1998. Relativistic Quatum Mechanics, Cambridge, United Kingdom, 594 pp.

4. Cashing, James T. 1998. Philosophical Concepts in Physics, Cambridge University Press, Cambridge, United Kingdom, 424 pp.

5. Eistein, Albert, 1971. La Relatividad. Editorial Grijalbo S. A. Mexico DF. Mexico, 202 pp.

6. Jackson, John David. 1962. Classical Electrodynamics. John Wiley and Sons. New York, USA, $848 \mathrm{pp}$.

7. Feyman, Richard P. 1985. QED, the strange theory of light and matter. Pricenton Univesity Press. New Jersey USA, 152 pp.

8. Tribble, Alan C. 1996. Princenton Guide to Advanced Physics. Pricenton Univesity Press. New Jersey USA, 327 pp.

9. Close, Frank. 1989. The quark struture of mater. En: Paul Davis (ed.) The New Physics, pp. 396-423. Cambridge Universtiy Press Cambridge, Cambridge, United Kingdom.

10. Lederman, Leon y Terisi Dick. 1993. The God Particle. New York USA, 434 pp.

11. Desloge, Edward A. 1966. Statistical Physics. Holt, Rinehart an Wiston, Inc. New York, USA, 437 pp.

12. Serway, Raymond A. Physics for Scientits and Engineers with Modern Physics, Saunders College Publishing, Fort Worth, USA, 1444 pp.

13. Quigg, Chris. 1983. Gauge of the strong, weak, and electromagnetic interactions. Addison Wesley Longman, Massachusetts, USA, 334pp.

14. Hooft, Gerard't. 1989 Gauge Theories of the Forces between Elementart Particles. En: Carrigan, Richrd A. y Trowes, W. Peter (ed.) Particle Physics in the Cosmos pp. 78-105, W.H. Freeman and Company, New York, USA.

15. Leyes de Conservación

16. Cahn, Robert N. y Goldhaber, Gerson 1989. The Experimental Foundations of Particles Physics, Cambridge Universtiy Press Cambridge, Cambridge, United Kingdom. 427 pp.

17. Strange, Paul. 1998 Relativistic Quantum Mechanics. Cambridge Universtiy Press Cambridge, Cambridge, United Kingdom. 594pp.

18. WA75 Collaboration. Charm production by $350 \mathrm{GeV} / \mathrm{c} \pi$ - interactions in nuclear emulsion. 
Prog. Theor. Phys. 87:1305-1314, 1992.

19. E653 Collaboration. Charm meson production in $850 \mathrm{GeV} / \mathrm{c}$ proton-emulsion interactions. Phys. Lett. B263: 573-578, 1991.

20. E691 Collaboration. Charmed meson decay results from Fermilab E691. AIP Conf. Proc. 243: 670-675, 1992.

21. Focus Collaboration (J.M. Link et al) Čerenkov Particles Idetification in FOCUS, internal memo, 2001

22. Rivera, Carlos. 1998. Nuevos Aspectos del Análisis de los Datos de Čerenkov del Experimento 831 de Fermilab. M.S. Tesis. Universidad de Puerto Rico, Recinto Universitario de Mayagüez. Mayagüez, Puerto Rico.

23. Montiel Peña, Enrigue. 1997. Gatillo interior de muones para E831. M.S. Tesis. Universidad de Puerto Rico, Recinto Universitario de Mayagüez, Mayagüez, Puerto Rico.

24. Focus Collaboration (J.M. Link et al.) The Target Silicon Detector for the Focus Spectometer. Internal memo, 2002.

25. Bediaga Ignacio and Gödel Carla. 1995. Introduction to Charm Decay Analysis in Fixed Target Experiments. Web site.....

26. Grim, Gray P. 1995. Doubly Cabbibo Suppressed D+ Decays and Singly Cabibbo Suppressed $D_{s}$ in $220 \mathrm{GeV} \gamma+p$ interactions. Thesis Ph.D. University of California, Davis, California, USA

27. Link, Jonathan Marion. 2001. Study of the Decay $D^{0} \rightarrow K \pi$ in FOCUS. Thesis Ph.D. University of California Davis, California, USA.

28. Cinquinni, Luca. 1996 Analysis of Charm Meson Semileptonic Decays and Carhm Baryon High Mass States. Thesis Ph.D. University of Colorado, Colorado, USA.

29. Cawlfield, Chistopher Alan. 2001. Studies of the semileptonic decays of D mesons. Thesis Ph.D. University of Illinois at Urbana-Champaing, Illinois, USA.

30. Vaandering, Eric W. 2000. Mass and Width Measurements of $\Sigma_{c}$ Baryons. Thesis Ph.D. University of Colorado, Colorado USA.

31. Johns, Will E. 1995. Measurments of the Semileptonic Decay of the Neutral Charmed Meson $D^{0} \rightarrow K \mu \nu$. University of Colorado, Colorado, USA.

32. Focus Collaboration (J.M. Link et al) Čerenkov Particles Indentification in FOCUS. 2001

33. Spiegel, Murray R. 1991. Estadística. Mc Graw Hill Book Company. New York USA. 556 pp. 
34. Holman, J.R. 1984. Experimental methods for engineers. Mc Graw Hill Book Company. New York USA 514 pp.

35. Spiegel, Murray R. 1991. Manual de fórmulas y Tablas de matemáticas. Mc Graw Hill Book Company. New York USA. 271 pp. 